\title{
Four-Scroll Hyperchaotic Attractor in a Five-Dimensional Memristive Wien Bridge Oscillator: Analysis and Digital Electronic Implementation
}

\author{
Gabin Jeatsa Kitio, ${ }^{1,2}$ Cyrille Ainamon, ${ }^{3}$ Karthikeyan Rajagopal ${ }^{4},{ }^{4}$ \\ Léandre Kamdjeu Kengne $\left(\mathbb{D},{ }^{1,2}\right.$ Sifeu Takougang Kingni ${ }^{\circ}{ }^{5}$, \\ and Justin Roger Mboupda Pone $\mathbb{1}^{2}$ \\ ${ }^{1}$ Research Unit of Condensed Matter, Electronics and Signal Processing (RU-MACETS), Department of Physics, \\ Faculty of Science, University of Dschang, P.O. Box 67, Dschang, Cameroon \\ ${ }^{2}$ Research Unit of Automation and Applied Computer (RU-AIA), Electrical Engineering Department of IUT-FV of Bandjoun, \\ University of Dschang, P.O. Box 134, Bandjoun, Dschang, Cameroon \\ ${ }^{3}$ Institut de Mathématiques et de Sciences Physiques, Université d'Abomey-Calavi, B.P. 613, Porto Novo, Benin \\ ${ }^{4}$ Center for Nonlinear Systems, Chennai Institute of Technology, Chennai, India \\ ${ }^{5}$ Department of Mechanical, Petroleum and Gas Engineering, Faculty of Mines and Petroleum Industries, University of Maroua, \\ P.O. Box 46, Maroua, Cameroon
}

Correspondence should be addressed to Justin Roger Mboupda Pone; pone00@yahoo.com

Received 24 July 2021; Accepted 1 October 2021; Published 19 October 2021

Academic Editor: Giuseppe Grassi

Copyright (c) 2021 Gabin Jeatsa Kitio et al. This is an open access article distributed under the Creative Commons Attribution License, which permits unrestricted use, distribution, and reproduction in any medium, provided the original work is properly cited.

\begin{abstract}
An electronic implementation of a novel Wien bridge oscillation with antiparallel diodes is proposed in this paper. As a result, we show by using classical nonlinear dynamic tools like bifurcation diagrams, Lyapunov exponent plots, phase portraits, power density spectra graphs, time series, and basin of attraction that the oscillator transition to chaos is operated by intermittency and interior crisis. Some interesting behaviors are found, namely, multistability, hyperchaos, transient chaos, and bursting oscillations. In comparison with some memristor-based oscillators, the plethora of dynamics found in this circuit with current-voltage (i-v) characteristic of diodes mounted in the antiparallel direction represents a major advance in the knowledge of the behavior of this circuit. A suitable microcontroller based design is built to support the numerical findings as these experimental results are in good agreement.
\end{abstract}

\section{Introduction}

An evidence fact in the research community is that the electronic circuits containing nonlinear elements exhibit rich dynamic behavior and it has been described in numerous books [1-4]. The research of chaotic memristive circuits is a hot topic of academic research in these recent years [5-8] due to their tremendous engineering applications. We can cite the field applications of communication systems, neural networks, image security, and so on. Memristor-based circuits are famous for displaying a rich variety of behaviors, including multiperiodic, quasiperiodic, and chaotic oscillations as well as self-pulsing and the coexistence of multiple attractors and hidden attractors [9-14]. The Wien bridge oscillator among many types of memristor-based oscillators appears to be one of the most studied recently with good standing papers published [15-17]. Memristor-based circuits are famous for displaying a rich variety of behaviors. These striking scenarios are defined as follows.

(i) Multistability is a critical property of nonlinear dynamical systems, where a variety of behaviors such as coexisting attractors can appear for the same parameters, but different initial conditions. The flexibility in the system's performance can be 
achieved without changing parameters. This striking scenario has been witnessed in numerous fields of engineering ranging across physics [18], biology [19], chemistry [20], electronics [21-23], and mechanics, as well as reported applications in oscillators and secure communications.

(ii) Quasiperiodicity is the property of a system that displays irregular periodicity [24]. Quasiperiodic behavior is a pattern of recurrence with a component of unpredictability that does not lend itself to precise measurement. It has been witnessed in rare systems such as the acoustic field [25], laser [26], and neural network [27].

(iii) Hyperchaotic scenario in the dynamical system is defined as a chaotic system with more than one positive Lyapunov exponent; this implies that its chaotic dynamics extend in several different directions simultaneously [24]. Therefore, comparing with the traditional chaotic system, the hyperchaotic system has more complex dynamical behaviors which can be used to enhance the security of the chaotic communication system [28]. Consequently, the topic of theoretical design and circuitry realization of various hyperchaotic systems has recently become a hotspot in the nonlinear research field. Hyperchaos has been found numerically and experimentally such as Chua's circuit [29], Chen system [30], or Lorenz equation [31].

(iv) Bursting oscillations are defined as complex oscillations consisting of spiking (cluster of spikes or rapid oscillations) separated by periods of relative quiescence [32]. They have been observed in many practical systems and found a multitude of applications in areas such as electromechanics [33], electronics [34], biology [35], and bioengineering of artificial organs [36]. They have been discovered in many fields, magnetohydrodynamics [37], plasma confinement [25], and X-ray pulsar emission [26]. In biological neurons and cells electrophysiology, bursting oscillations play an important role in information processing. Moreover, in biological neurons, bursting oscillations are important for motor pattern generation and synchronization.

(v) Transient chaos is a dynamical behavior that displays the existence of chaotic behavior on finite time [38]. Generally, the phenomenon of transient chaos can be observed in dynamical system with boundary crisis [39] and also in families of the logistic and Hénon maps.

Zhijun and Yicheng [40] employed a piecewise linear memristor to construct a fourth-order memristor-based Wien bridge circuit with hyperchaotic dynamics. Wu et al. [41] constructed an active generalized memristor, in which a fourthorder Wien bridge chaotic oscillator was designed further. In recent years, the electronic research team focused on the infinitely differentiable characteristic equation of the diode component in electronic chaotic circuits replacing nonsmooth ones. The synthesis are well presented in $[42,43]$ just to name some well-standing papers. Some rare and interesting dynamics are found, namely, coexisting hidden attractors, quasi periodicity with antimonotonicity, and hyperchaos. As we recall, finding chaotic circuits, (i) which modeled some important unsolved problems in nature, (ii) shed insight on that problems, and (iii) exhibited some behavior previously unobserved [44], is still a major interest. For this purpose, we explore the 5D Wien bridge memristive oscillator with antiparallel diodes with smooth (i-v) characteristics not yet explored in this circuit with interesting dynamics discover:

(i) Intermittency route to chaos

(ii) Transient chaos

(iii) Hyperchaos with offset boosting and partial amplitude control

(iv) Multistability

(v) Bursting oscillations

(vi) The successful microcontroller implementation

The (i-v) characteristic model without approximations of the behavior of the nonlinear element diodes connected in antiparallel direction, therefore, constitutes an advance in the field of research for this Wien bridge oscillator.

The rest of this paper is organized as follows. In Section 2 , the model and analysis of a memristive Wien bridge oscillator are presented. It is followed in Section 3 by the numerical analyses highlighting transitions to chaos. Then, in Section 4, some complex dynamics are discovered in this oscillator. We then continue with the microcontroller implementation in Section 5 to verify the numerical findings. The paper ends with some concluding remarks.

\section{Modelling and Analysis of a Memristive Wien Bridge Oscillator}

The schematic diagram of a memristive circuit based on the 5D Wien bridge oscillator is presented in Figure 1.

2.1. The Model of the Circuit. The circuit of Figure 1 consists of three capacitors $C_{1}, C_{2}, C_{3}$; an inductor $L_{1}$ with its internal resistor $R_{1}$; an operational amplifier; two antiparallel diodes $D_{1}$, $D_{2}$; three resistors $R_{2}, R_{3}, R_{4}$; and a flux-controlled memristor $\omega(\varphi)$. The authors in $[16,45]$ used a piecewise linear function to describe the voltage-current characteristics of two antiparallel diodes. The exponent of the internal state of the fluxcontrolled memristor was set to first order as in [46]. In this work, the current-voltage characteristic of two antiparallel diodes $D_{1}$ and $D_{2}$ is described without any approximation:

$$
i_{d}=i_{S}\left(e^{\rho\left(u_{1}-u_{3}\right)}-1\right)-i_{S}\left(e^{-\rho\left(u_{1}-u_{3}\right)}-1\right)=2 i_{S} \sinh \left(\rho \left(u_{1}\right.\right.
$$
$\left.\left.-u_{3}\right)\right)$, where $\rho=1 /\left(\eta V_{T}\right)$, the parameters $i_{s}=2.682 \mathrm{nA}$, and $V_{T}=26 \mathrm{mV}$ stand for the reverse saturation current, ideality factor, and the thermal voltage of the diodes, respectively. The exponent of the internal state of the memristor here is set to second order as in [47]. The five dynamic 


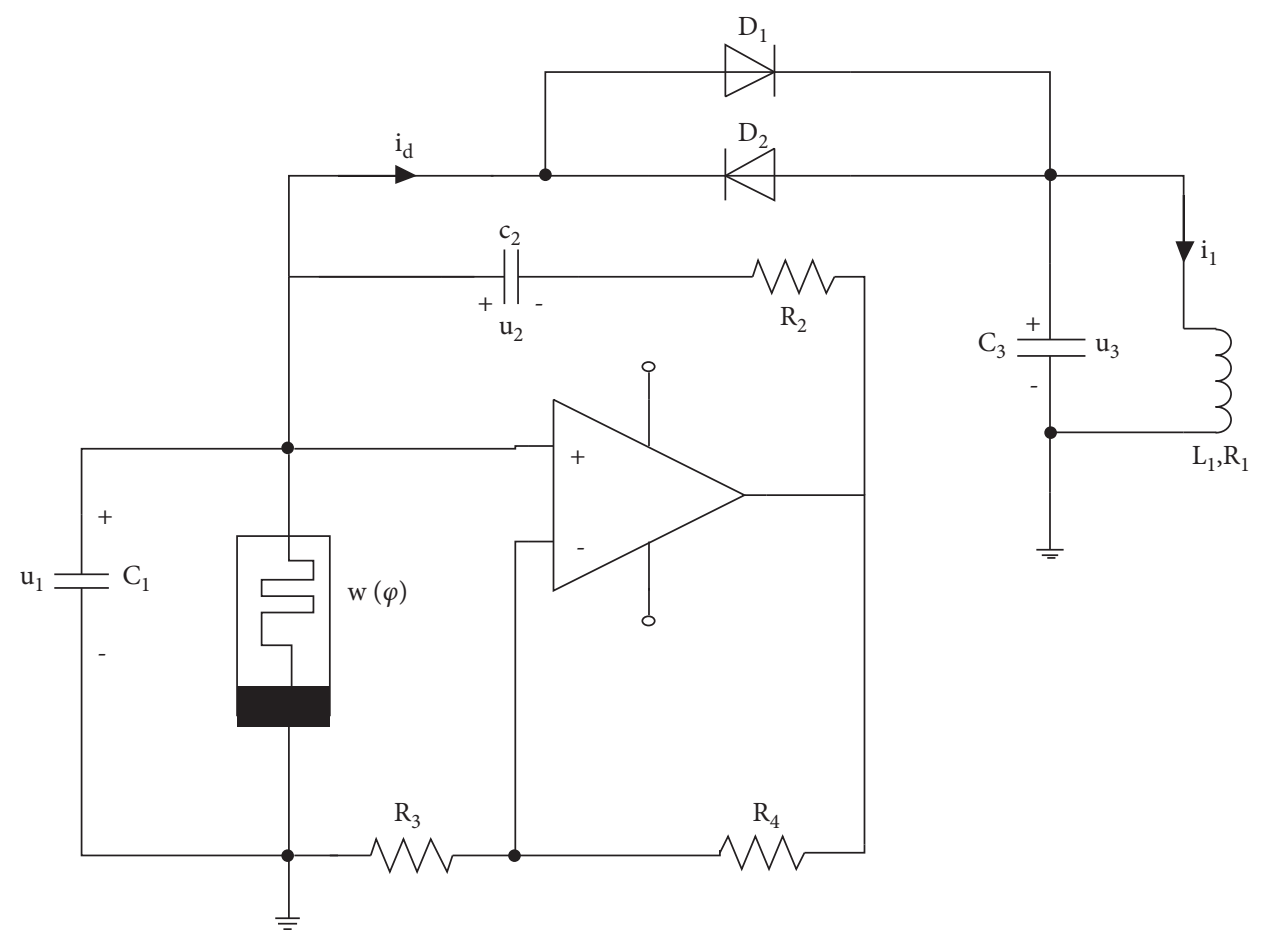

Figure 1: The memristive Wien bridge circuit.

elements $C_{1}, C_{2}, C_{3}, L$, and memristor correspond to each state variable $u_{1}, u_{2}, u_{3}, i_{1}$, and $\phi$, respectively. For analysis of the circuit, Kirchhoff's law is applied to the circuit of Figure 1 to reveal five sets of first-order differential equations:

$$
C_{1} \frac{\mathrm{d} u_{1}}{\mathrm{~d} t^{\prime}}=\frac{R_{4}}{R_{2} R_{3}} u_{1}-w(\phi) u_{1}-\frac{1}{R_{2}} u_{2}-2 i_{S} \sinh \left(\rho\left(u_{1}-u_{3}\right)\right),
$$

$$
\begin{aligned}
C_{2} \frac{\mathrm{d} u_{2}}{\mathrm{~d} t^{\prime}} & =\frac{R_{4}}{R_{2} R_{3}} u_{1}-\frac{1}{R_{2}} u_{2}, \\
C_{3} \frac{\mathrm{d} u_{3}}{\mathrm{~d} t^{\prime}} & =2 i_{S} \sinh \left(\rho\left(u_{1}-u_{3}\right)\right)-i_{1}, \\
L_{1} \frac{\mathrm{d} i_{1}}{\mathrm{~d} t^{\prime}} & =u_{3}-R_{L} i_{1}, \\
\frac{\mathrm{d} \phi}{\mathrm{d} t^{\prime}} & =-u_{1}-\alpha \phi+u_{1}^{2} \phi,
\end{aligned}
$$

where the parameter $\alpha$ is associated with the memristor and $w(\phi)=\left(a+3 b^{\prime} \phi^{2}\right)$ is the memristance. Let us define

$$
x=u_{1} / \rho, \quad y=u_{2} / \rho, \quad z=u_{3} / \rho, \quad w=i_{1} \sqrt{R_{2} R_{3}} / \rho,
$$
$v=\phi /\left(\rho C_{1} \sqrt{R_{2} R_{3}}\right) t=t^{\prime} / C_{1} \sqrt{R_{2} R_{3}}, \quad b=R_{2} R_{3}\left(C_{1} \rho\right)^{2} b^{\prime}$, $c=R_{4} / \sqrt{R_{2} R_{3}}, d=\sqrt{R_{3} / R_{2}}, e=\sqrt{R_{2} R_{3}} f=\rho C_{1}, g=2 i_{s} / \rho$, $k=R_{2} R_{3} C_{1} / L, \delta=\rho^{2}, C_{1}=C_{2}=C_{3}$ by inserting the normalized parameters in the set of equations (1a)-(1e); the dimensionless set of equations (1a)-(1e) is given by equations $(2 \mathrm{a})-(2 \mathrm{e})$ suitable for numerical studies:

$$
\begin{aligned}
\frac{\mathrm{d} x}{\mathrm{~d} t} & =\left[c-e\left(a+3 b v^{2}\right)\right] x-d y-e g \sinh (x-z), \\
\frac{\mathrm{d} y}{\mathrm{~d} t} & =c x-d y, \\
\frac{\mathrm{d} z}{\mathrm{~d} t} & =e g \sinh (x-z)-w, \\
\frac{\mathrm{d} w}{\mathrm{~d} t} & =k z, \\
\frac{\mathrm{d} v}{\mathrm{~d} t} & =-x+e f\left(-\alpha+\delta x^{2}\right) v .
\end{aligned}
$$

2.2. Mathematical Analysis. System (2a)-(2e) is invariant under the transformation: $S(x, y, z, w, v) \longrightarrow S(-x,-y$, $-z,-w,-v)$; therefore, system (2a)-(2e) is symmetry about the origin. The origin of the state space is a trivial equilibrium point $E(0,0,0,0,0)$ meaning that the solution shows twin symmetric around the origin. The other equilibrium points of system (2a)-(2e) are obtained by solving $\mathrm{d} x / \mathrm{d} t=0, \mathrm{~d} y / \mathrm{d} t=0, \mathrm{~d} z / \mathrm{d} t=0, \mathrm{~d} w / \mathrm{d} t=0, \mathrm{~d} v / \mathrm{d} t=0$, which gives

$$
\begin{aligned}
& z^{*}=0, \\
& y^{*}=\frac{c x^{*}}{d}, \\
& w^{*}=e g \sinh \left(x^{*}\right),
\end{aligned}
$$




$$
\left[a+\frac{3 b\left(x^{*}\right)^{2}}{(e f)^{2}\left[-\alpha+\delta\left(x^{*}\right)^{2}\right]^{2}}\right] x^{*}+g \sinh \left(x^{*}\right)=0 .
$$

Equation (3d) cannot be solved analytically. Then, the Newton-Raphson method [48] is used to find the value of $x^{*}$ for the chosen value $a=0.05, b=0.03, f=1, g=1, \alpha=2$, delta $=1, e=2$, and the method yield $x^{*}=-3.08 e-18$ resulting in the trivial $E$. By linearizing system (2a)-(2e) around $E$, we obtain

$$
J a c=\left(\begin{array}{ccccc}
{\left[c-e\left(a+3 b v^{2}\right)\right]-e g \cosh (x-z)} & -d & e g \cosh (x-z) & 0 & -6 e b v x \\
c & -d & 0 & 0 & 0 \\
e g \cosh (x-z) & 0 & -e g \cosh (x-z) & -1 & 0 \\
0 & 0 & k & 0 & 0 \\
(-1+2 e f \delta x v) & 0 & 0 & 0 & e f\left(-\alpha+\delta x^{2}\right)
\end{array}\right)
$$

where Jac is the Jacobian matrix. Thus, the stability of $E$ can be determined by solving the characteristic equation $\operatorname{det}\left(M_{j}-\lambda I_{5}\right)=0$, where $I_{5}$ represents the $5 \times 5$ identity matrix. Table 1 illustrates the eigenvalues obtained by the Newton-Raphson method.

The screening parameter $\alpha$ is kept in the range $1.4<\alpha<2$ while the other parameters of the model are the ones defined previously.

We can conclude that, for $\alpha$ belonging to the interval, the system can develop self-excited attractors.

The overview of the stability of system $(2 a)-(2 e)$ is performed by plotting the stability diagram versus parameters $a$ and $\alpha$.

In Figure 2, we can notice that the unstable area is limited to the stable area at a critical value of $a$ around 0.75 ; this helps to choose the value of the parameter for numerical analyses.

\section{Numerical Analysis}

3.1. 2D Bifurcation Diagrams. Numerical analyses of the dynamical system can be obtained by plotting the 2-D MLE when varying simultaneously two parameters to provide global information about the dynamic behavior of the system under investigation [49].

The colors on these diagrams of the model of our oscillator vary according to the value of the MLE computed using the well-known method of Wolf et al. [50]. In these figures, the light green, cyan, and magenta characterize a chaotic motion while the dark-green yellow and dark red represent periodic or quasiperiodic motion. It is therefore visible that parameters $c, \mathrm{~d}$, and $g$ provide many diverse dynamics in contrary to the parameter $e$ that is monotone. For these reasons, we choose them in their interesting interval to study the scenario toward chaos (Figure 3).

3.2. Transitions to Chaos. In this section, intensive numerical analyses are performed by monitoring the bifurcation parameter $\alpha$ and initial states. We plot the local maxima of the coordinate $x\left(x_{\max }\right)$ and record the Lyapunov spectra. We noticed sparse chaotic windows alternating with periodic ones while increasing or decreasing the bifurcation parameter $\alpha$. The red curve is obtained during the increasing path of $\alpha$ while the red one is during the decreasing path.

In this manner, the hysteresis firstly discovered in dynamical systems by Berglund [51] occurs in Figure 4(a) while the red and black curve does not overlap. It is used here to discover symmetric or multiple attractors.

The chaos behavior is obtained as we can see by the intermittency route. We note that this phenomenon is rarely found in dynamical system interring chaotic dynamics [52]. It is described as a scenario involving several frequencies and spontaneously becomes chaotic while varying the bifurcation parameter. The sudden changes in the quality behavior (competition between several frequencies and chaos) are revealed in detail in Figure 5 where some phase portraits with corresponding power density spectra are plotted.

According to Figure 5, we can reveal to the reader the transition to chaos (in forward and in the reversed directions of the bifurcation parameter $\alpha$ ) from Figure $5(\mathrm{j}) €$ Figure $5(\mathrm{k})$. We can observe a periodic behavior suddenly followed by chaotic dynamics by a tiny variation of $\alpha$.

In this numerical research, it is usually during the intermittency route to encounter the same scenario while time elapsed. In this situation, chaos can appear and disappear to become periodic: this scenario is called transient chaos and it is very rare in dynamical systems.

3.2.1. Transient Chaos Behavior. The findings of transients' chaos are of great interest since they are believed to be the culprit for disastrous such as voltage collapse in electric power systems [53] and species extinction in ecology. We choose the set of system parameters $a=0.05, b=0.03, c=6$, $d=3, e=2, f=1, g=1, k=2, \alpha=1.3$, delta $=1$ and plot the time trace of the dynamical system in Figure 6.

In Figure 6, one can notice that starting the system at $t=0$, the behavior of the system is chaotic until $t=600 \mathrm{~s}$. This description is revealed by the chaotic attractor in (b) with the corresponding power density spectra. Passing the critical time $600 \mathrm{~s}$, the system becomes regular as shown in Figure 6(d). This phenomenon is also reported in memristive systems [54] including Chua's [55] and Duffing oscillators [56] and deserves to be shared. 
TABLE 1: Eigenvalues and stability nature of the equilibrium point $E$ computed for some discrete values of the parameter $\alpha$.

\begin{tabular}{lcccccc}
\hline$\alpha$ & $\lambda_{1}$ & $\lambda_{2}$ & $\lambda_{3}$ & $\lambda_{4}$ & $\lambda_{5}$ & Stability nature of E \\
\hline 2 & -4.0000 & $0.8727+1.3475 i$ & $0.8727-1.3475 i$ & $-1.4227+1.6926 i$ & $-1.4227-1.6926 i$ & Instable \\
2.5 & -5.0000 & $0.8727+1.3475 i$ & $0.8727-1.3475 i$ & $-1.4227+1.6926 i$ & $-1.4227-1.6926 i$ & Instable \\
2.8 & -5.6000 & $0.8727+1.3475 i$ & $0.8727-1.3475 i$ & $-1.4227+1.6926 i$ & $-1.4227-1.6926 i$ & Instable \\
3 & -6.0000 & $0.8727+1.3475 i$ & $0.8727-1.3475 i$ & $-1.4227+1.6926 i$ & $-1.4227-1.6926 i$ & Instable \\
3.2 & -6.4000 & $0.8727+1.3475 i$ & $0.8727-1.3475 i$ & $-1.4227+1.6926 i$ & $-1.4227-1.6926 i$ & Instable \\
3.3 & -6.6000 & $0.8727+1.3475 i$ & $0.8727-1.3475 i$ & $-1.4227+1.6926 i$ & $-1.4227-1.6926 i$ & Instable \\
1 & -2.0000 & $0.8727+1.3475 i$ & $0.8727-1.3475 i$ & $-1.4227+1.6926 i$ & $-1.4227-1.6926 i$ & Instable \\
1.2 & -2.4000 & $0.8727+1.3475 i$ & $0.8727-1.3475 i$ & $-1.4227+1.6926 i$ & $-1.4227-1.6926 i$ & Instable \\
1.4 & -2.8000 & $0.8727+1.3475 i$ & $0.8727-1.3475 i$ & $-1.4227+1.6926 i$ & $-1.4227-1.6926 i$ & Instable \\
\hline
\end{tabular}

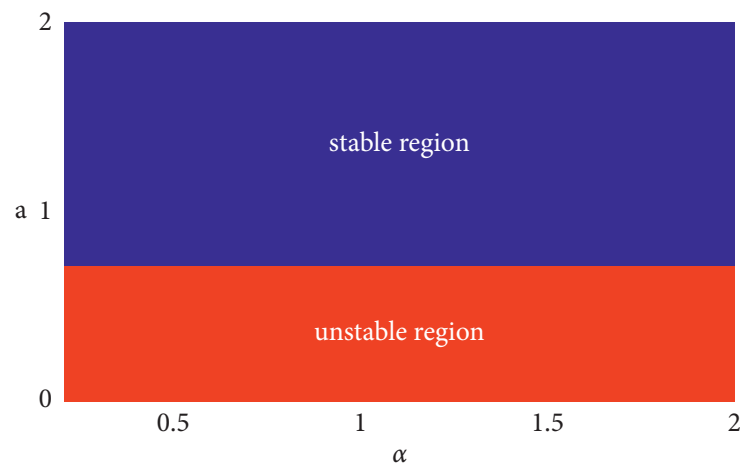

FIGURE 2: Stability diagram of the Wien bridge oscillator in the plane $(\alpha, a)$ highlighting the stable regions (blue) and unstable regions (red) of system (1a)-(1e).

3.2.2. Intermittency Route to Chaos during Symmetric Interior Crisis. Another interesting dynamic found in this system is the interior crisis. It describes the bifurcation events in which a chaotic attractor suddenly expands in size. It was initially observed by Grebori et al. [57].

In Figure 7, when the control parameter is varied, we noticed the expansion in size of the red and black attractors until they merged to form a unique huge attractor in Figure 7(f). This striking phenomenon is shown with the time traces of the black and red attractors in the right column of Figure 7.

The description of the multiple routes to chaos observed in system (2a)-(2e) rise to a very interesting and complex behavior as we revealed in the next section.

\subsection{Complex Dynamics of the Oscillator}

3.3.1. Multistability. The famous and interesting behavior of multistability in the dynamical system was shown in the optical chain by [58] and in the isolation of a new defect in $n$-type silicon $[59,60]$. It was recently encountered in well-known systems such as Chua ([61]) and Sprott [62], jerk system [63]. A simple technique to detect this property is to scan the bifurcation diagram upward and downward using the same control parameter (see Figure 4). In the light of this technique, one can obverse in Figure 4(a) some windows where the black curve and the red curve do not overlap showing the multistability phenomenon. We draw a zoom in the interesting interval of the control parameter to share this scenario, Figure 8(a).
In Figures 8(b1) and (b2), one can see that the 4 attractors coexist for the same set of system parameters. The red ones are obtained with positive initial conditions while black attractors are obtained with negative initial conditions. The basin of attraction showing the space of initial conditions resulting from each coexisting steady state is plotted in Figure 9. Recall that the basin of attraction associated with attractors red or black is the closure of the set of initial points that, taken as initial conditions, converge to red or black attractors when time increases to infinity. This subset-plane of initial points are determined using the computation of maximum Lyapunov Exponent (MLE) using the well-known algorithm by Wolf et al. [50]. For the 5D system under study, we fixed the initial points $x_{2}(0)=x_{3}(0)=x_{4}(0)=1$. The system parameters are set in the caption of Figure 9 and remain unchanged during the computation. For any couple of starting points.

$-4<x_{1}(0)<4$ and $-10<x_{5}(0)<10$, the long-term behavior of system (1a)-(1e) is computed using the Rung-Kutta algorithm, and the MLE is determined using the Wolf et al.'s method. Then, it is saved. If:

(a) MLE $>0$, we plot on the substate space the point with magenta color for positive chaotic attractors while the blue color area is for negative ones.

(b) $M L E \leq 0$, we plot on the substate space the point with black color (for negative limit cycles) and red color (for positive limit cycles) for initial conditions that led to periodic attractors.

In Figure 9, the reader can discover the fractal form of the substate space resulting in the complexity of system (1a)-(1e). Note that other planes are not plotted for simplicity purposes.

3.3.2. Hyperchaos. The new memristive Wien Bridge oscillator, as in Figure 1, generates hyperchaotic attractor with two positive Lyapunov exponents. In Table 2, Lyapunov exponents and dynamics of system (1a)-(1e) for different values of $\alpha$ are given.

Figure 10 displays the phase portraits of the hyperchaotic attractors.

As we can see, the spiraling trajectories are much denser than those in the chaotic ones, showing to the reader the striking phenomenon of hyperchaos. In Table 2, there are two Lyapunov exponents greater than zero. 


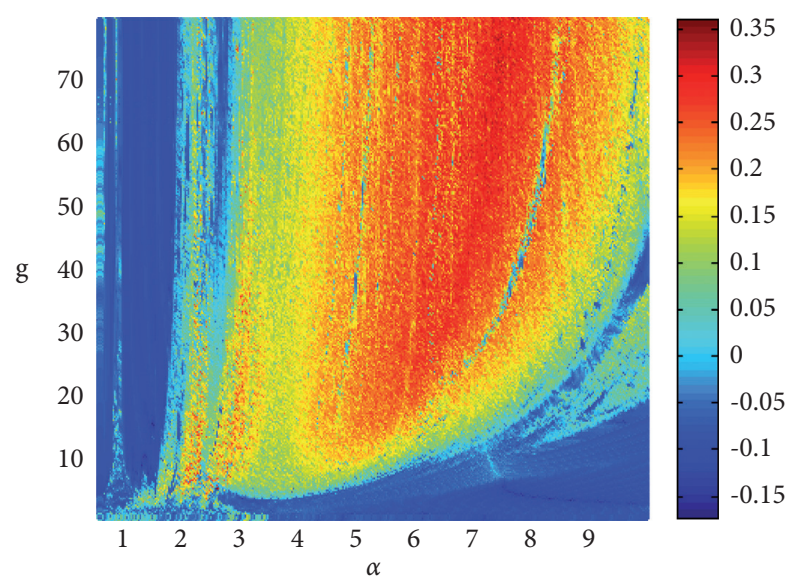

(a)

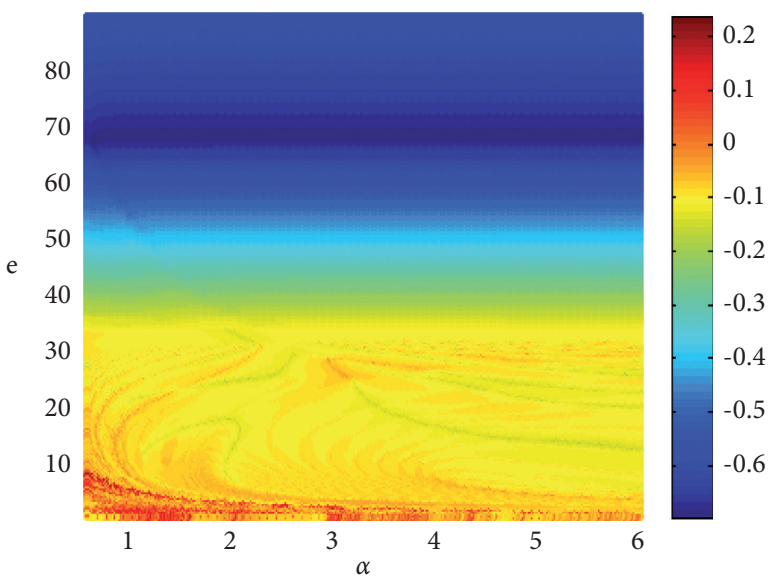

(c)

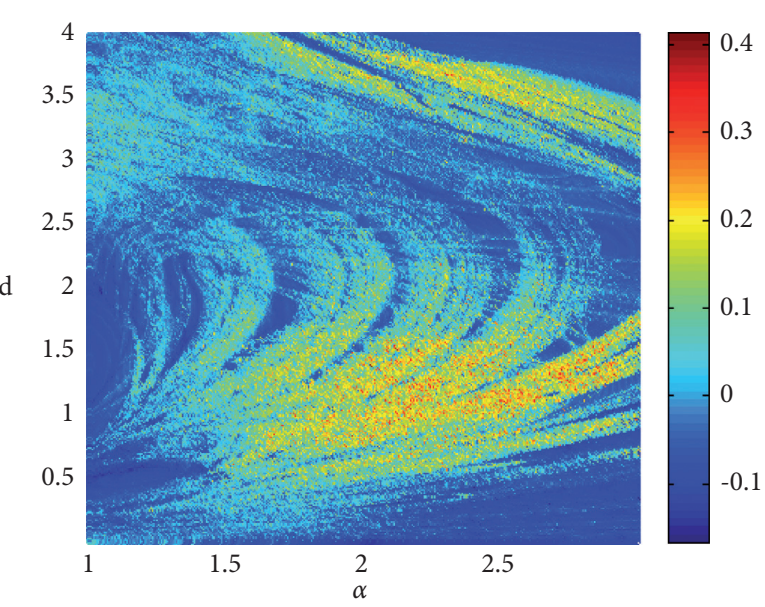

(b)

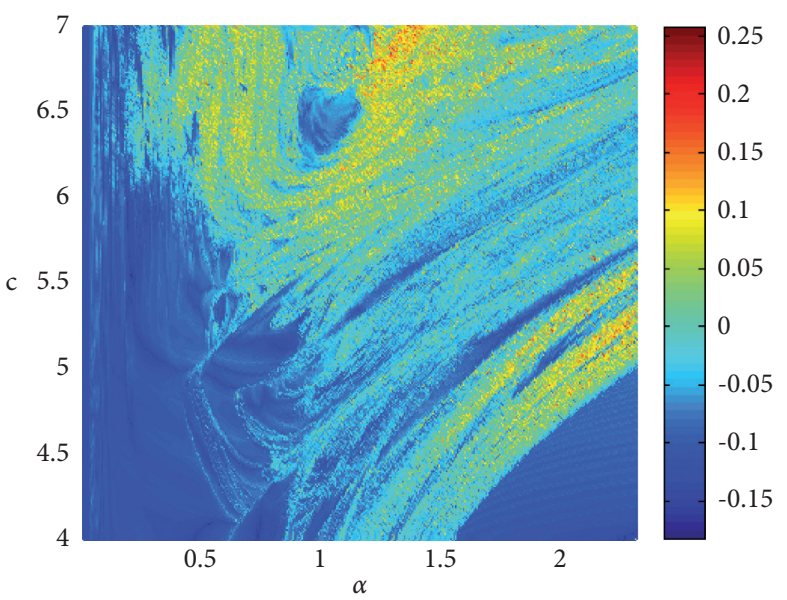

(d)

Figure 3: 2D maximum Lyapunov exponent (MLE) bifurcation diagrams in the plane: (a) $(g, \alpha)$; (b) $(d, \alpha)$; (c) $(d, c)$; and (d) $(\alpha, c)$ depicting the region of complex dynamics of system (2a)-(2e) with respect to the MLE (right column bar); the system parameters are: $a=0.05$; $b=0.03 ; c=6 ; d=3 ; e=2 ; g=1 ; k=2$. Initial conditions are $\left(x_{0} ; y_{0} ; z_{0} ; w_{0} ; v_{0}\right)=(1 ; 1 ; 1 ; 0.1 ; 1)$ (color figure online).

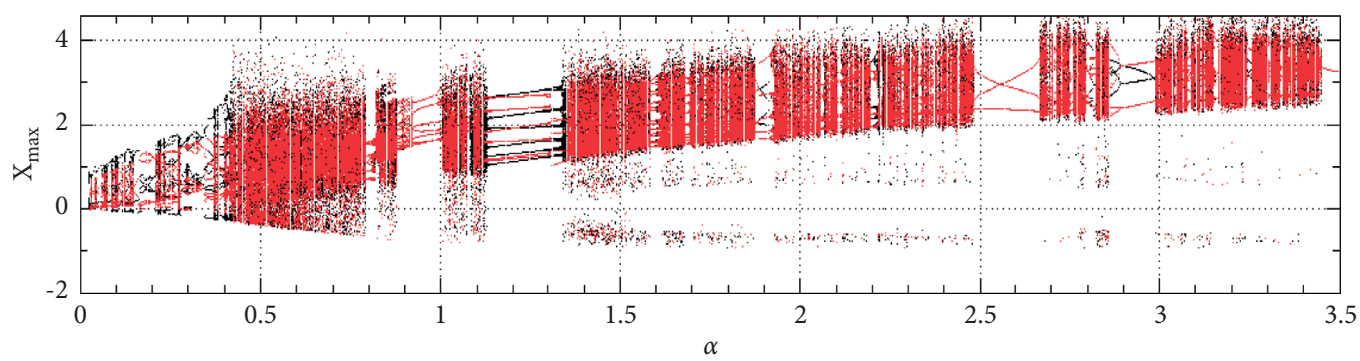

(a)

FIgURE 4: Continued. 

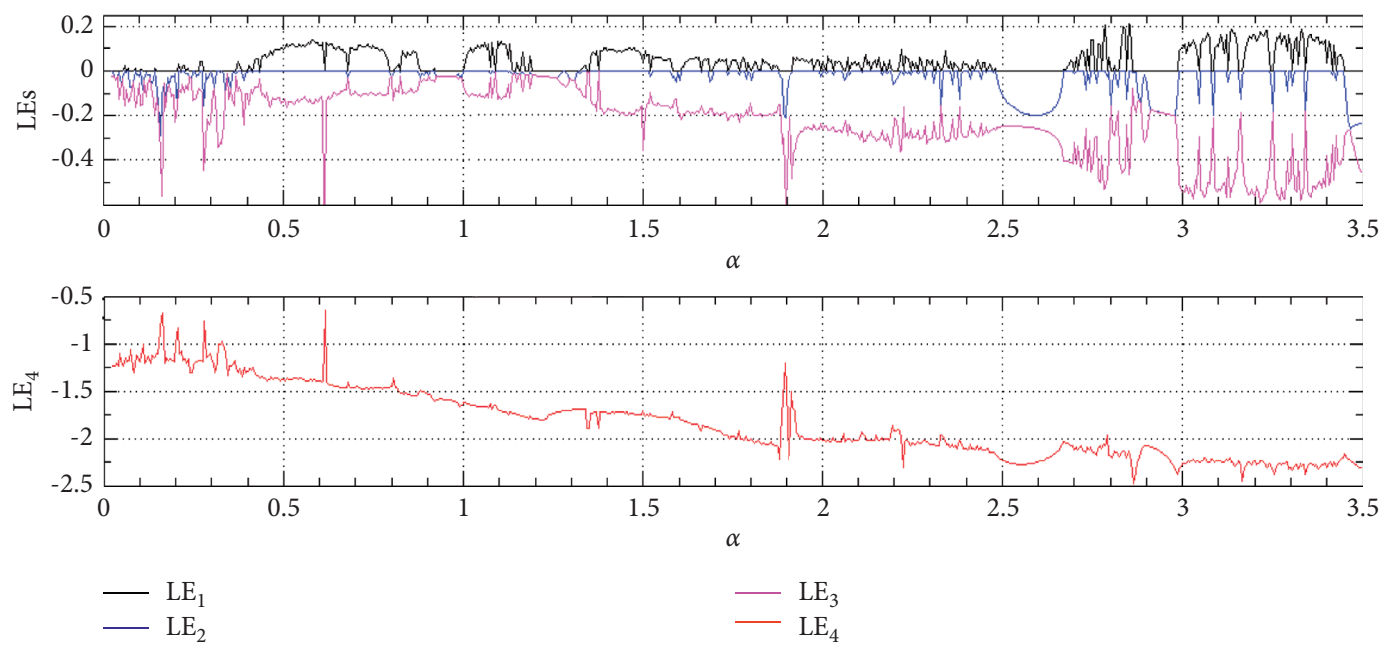

(b)

FIgURE 4: Bifurcation (2a)-(2e) of the system showing local maxima of the coordinate $x_{\max }$ versus the parameter $\alpha$ black curve for increasing parameter $\alpha$ and red for decreasing parameter $\alpha$. (b): the corresponding graphs of Lyapunov exponent spectra. The system parameters are $a=0.05, b=0.03, c=6, d=3, e=2, \mathrm{~g}=1, k=2$, and $f=1$. Initial conditions are $\left(x_{0} ; y_{0} ; z_{0} ; w_{0} ; v_{0}\right)=(1 ; 1 ; 1 ; 0.1 ; 1)$ (color figure online).
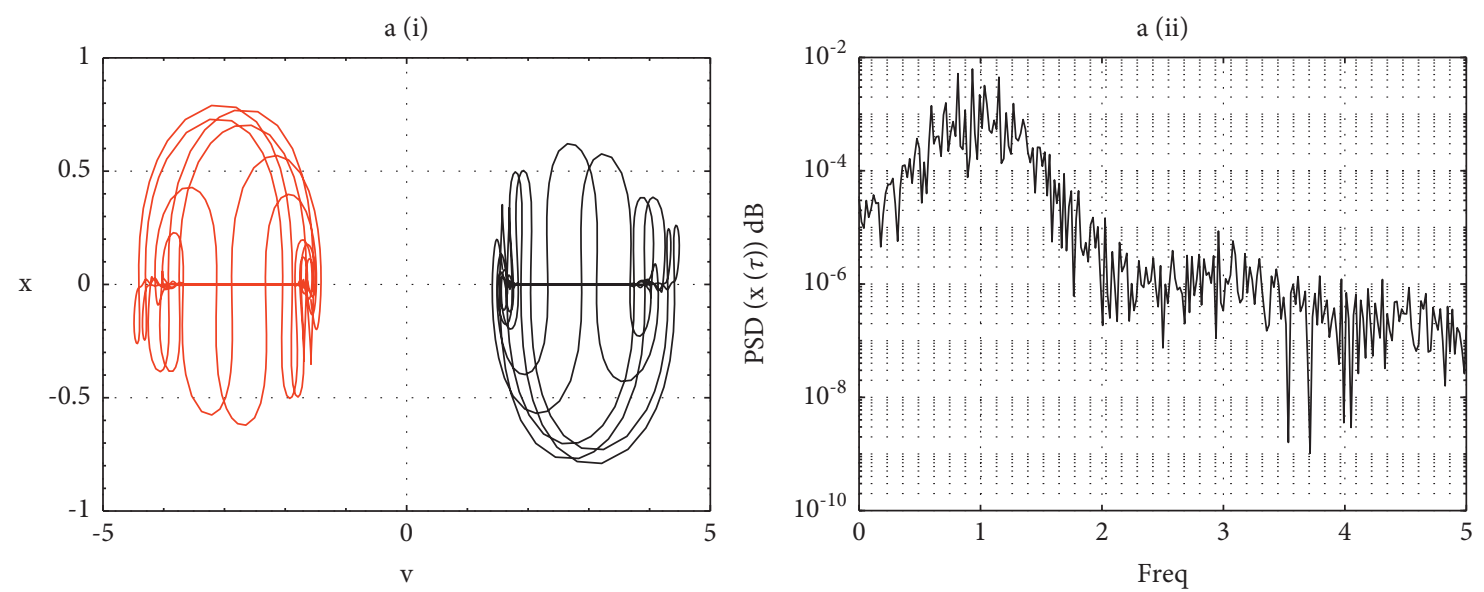

(a)
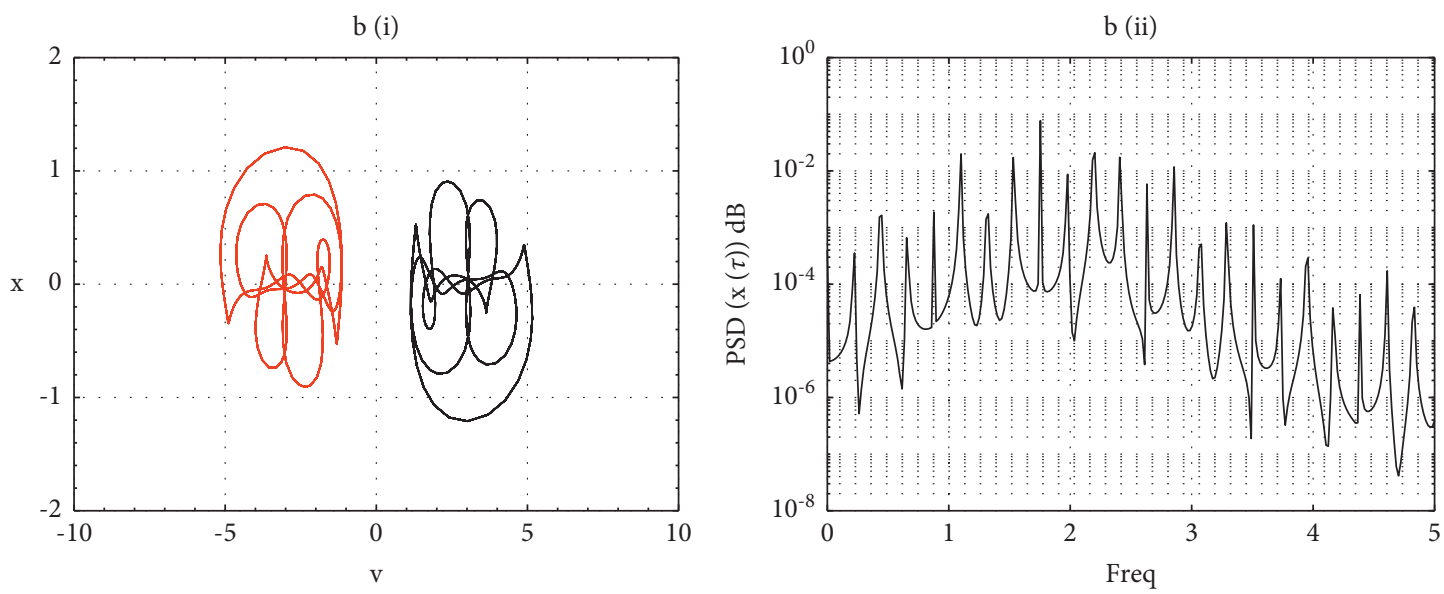

(b)

FIgURE 5: Continued. 

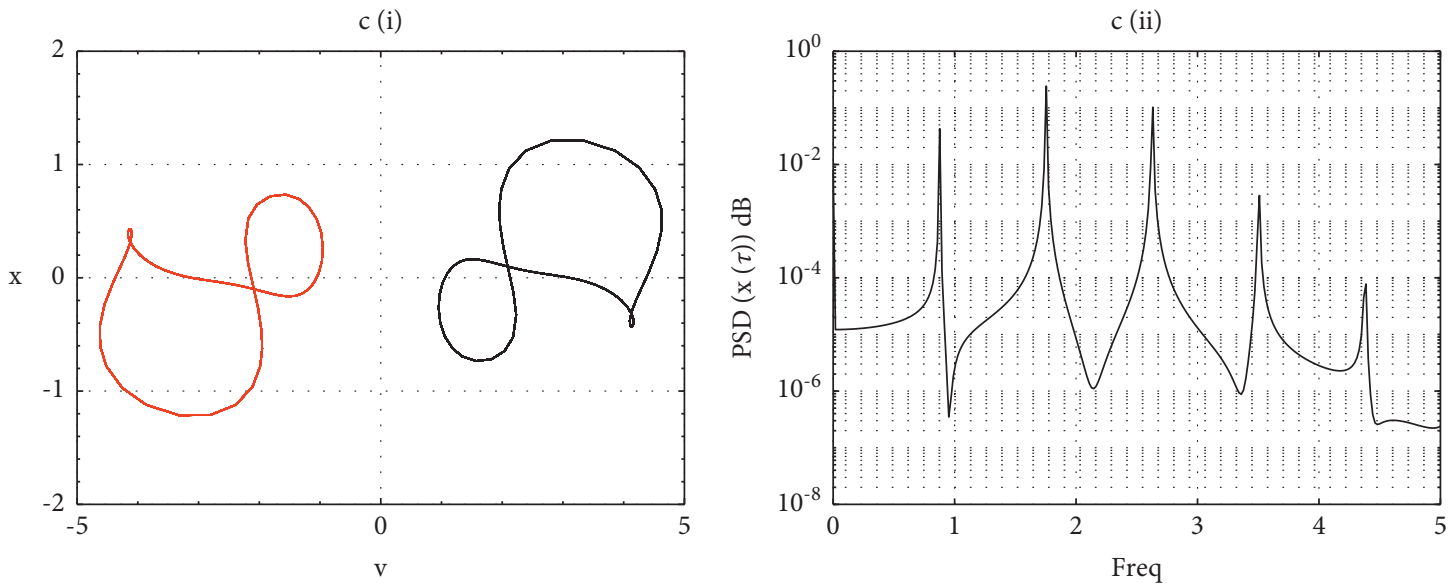

(c)
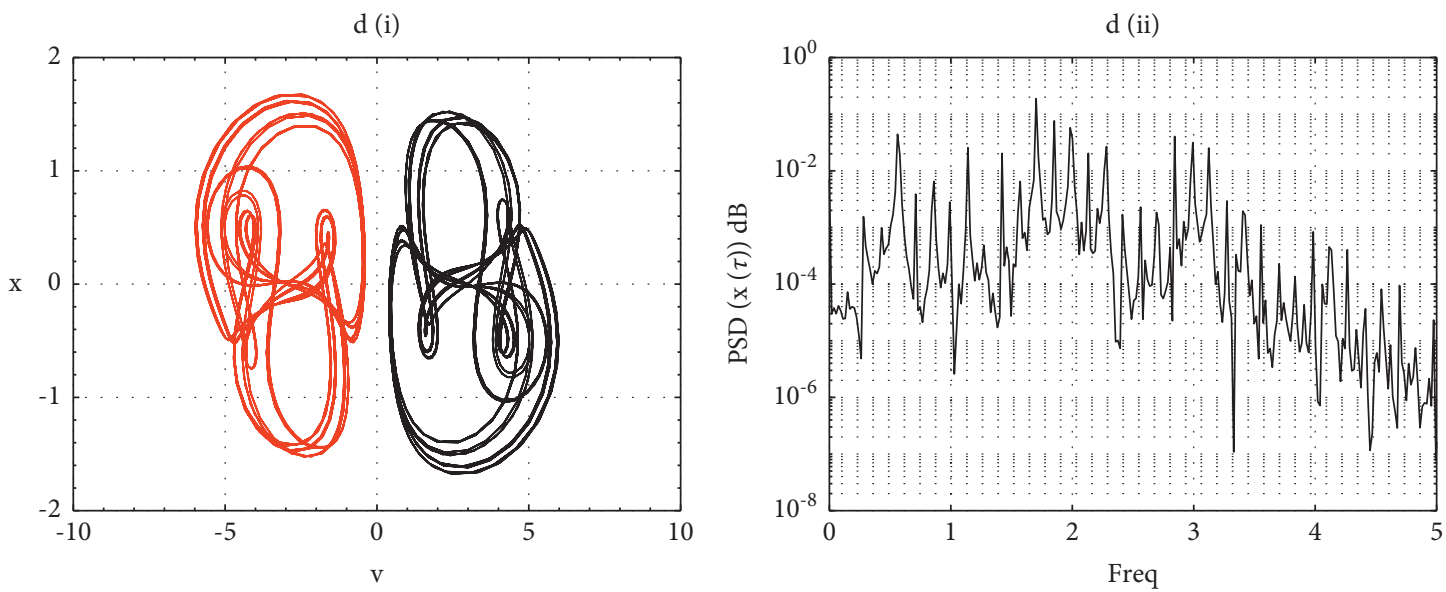

(d)
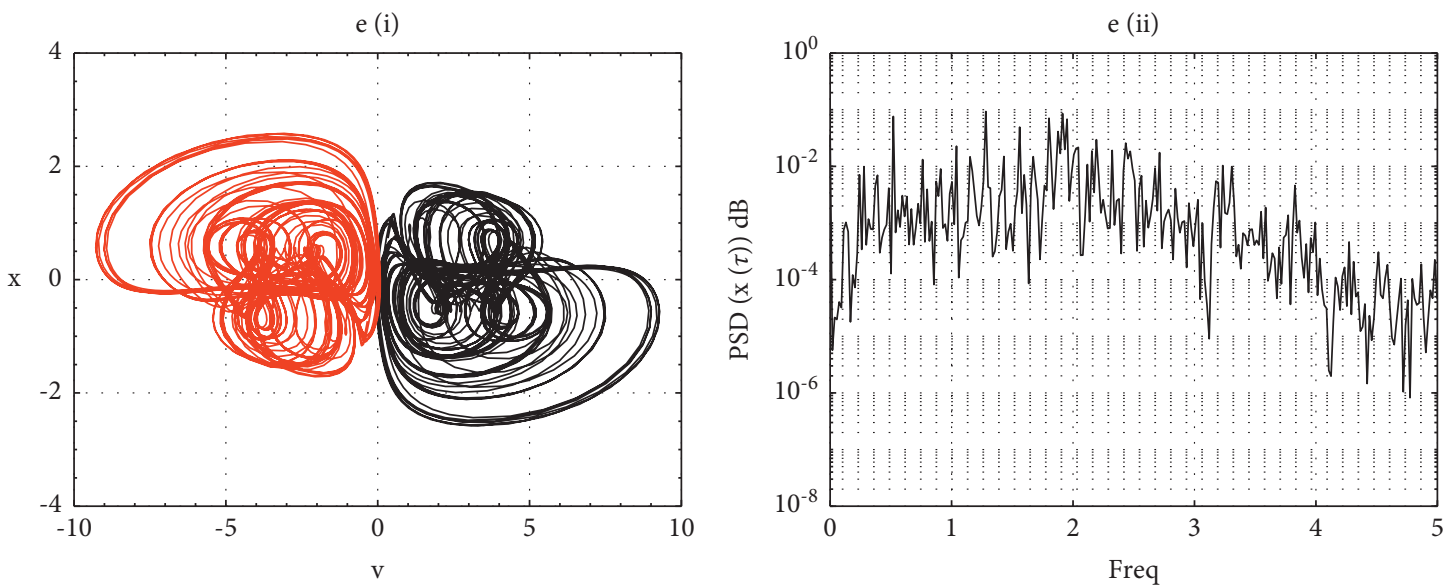

(e)

Figure 5: Continued. 

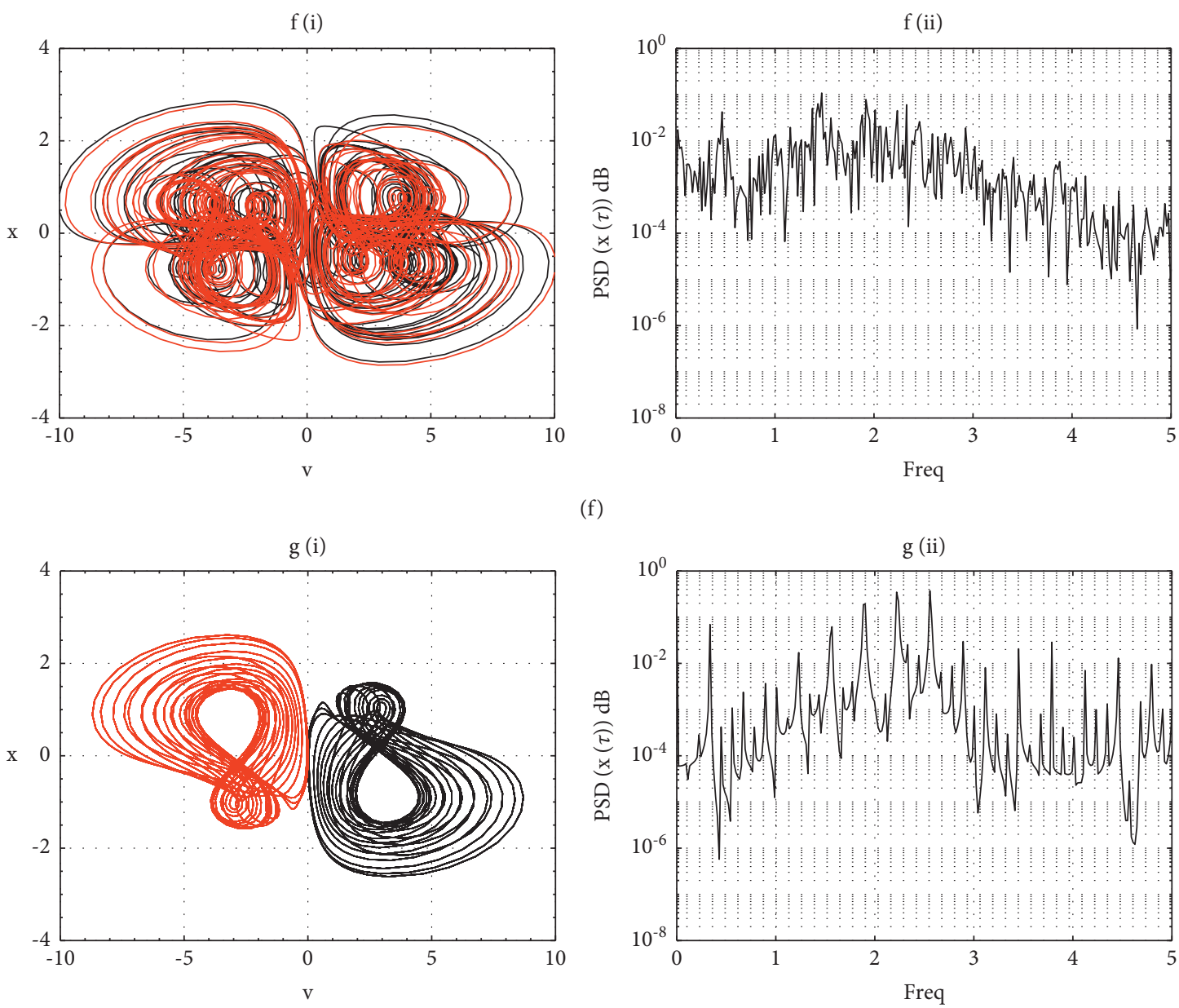

(f)

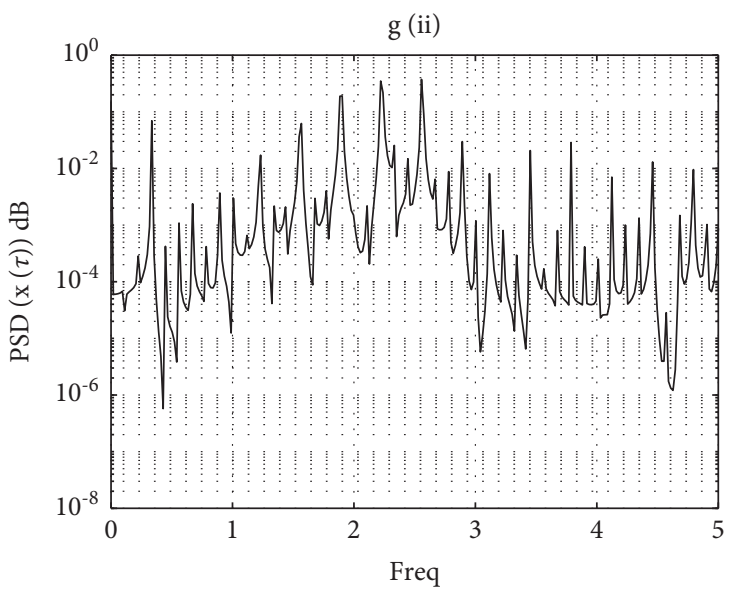

(g)
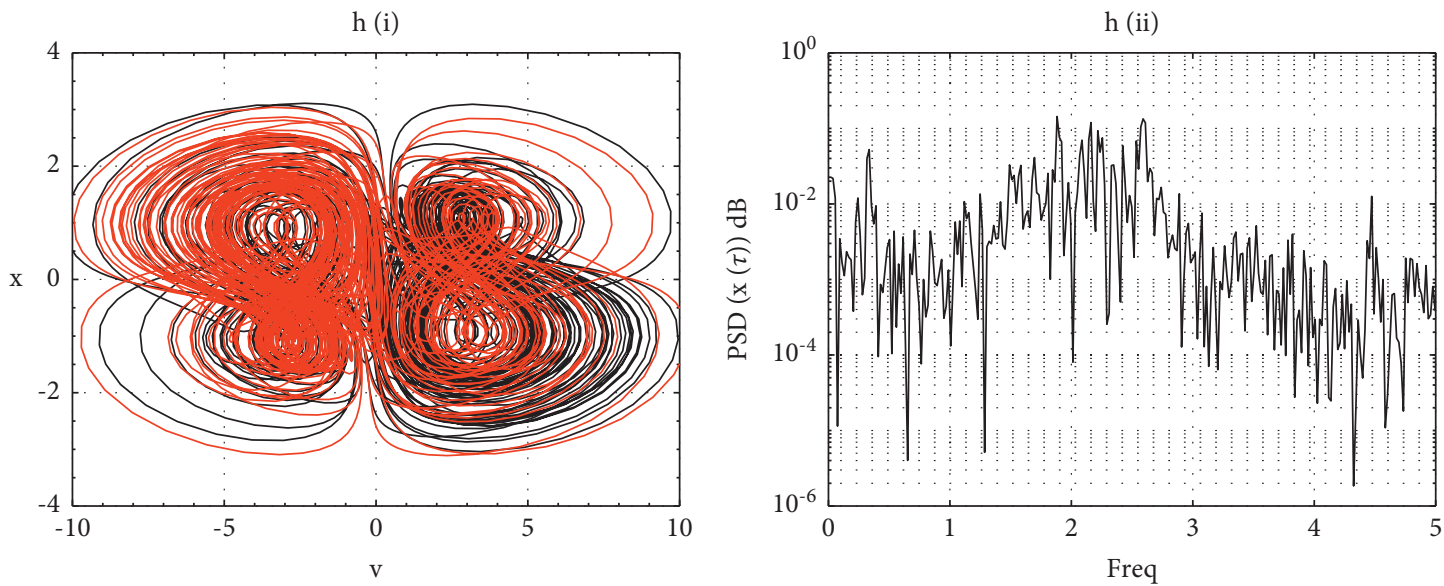

(h)

FIGURE 5: Continued. 

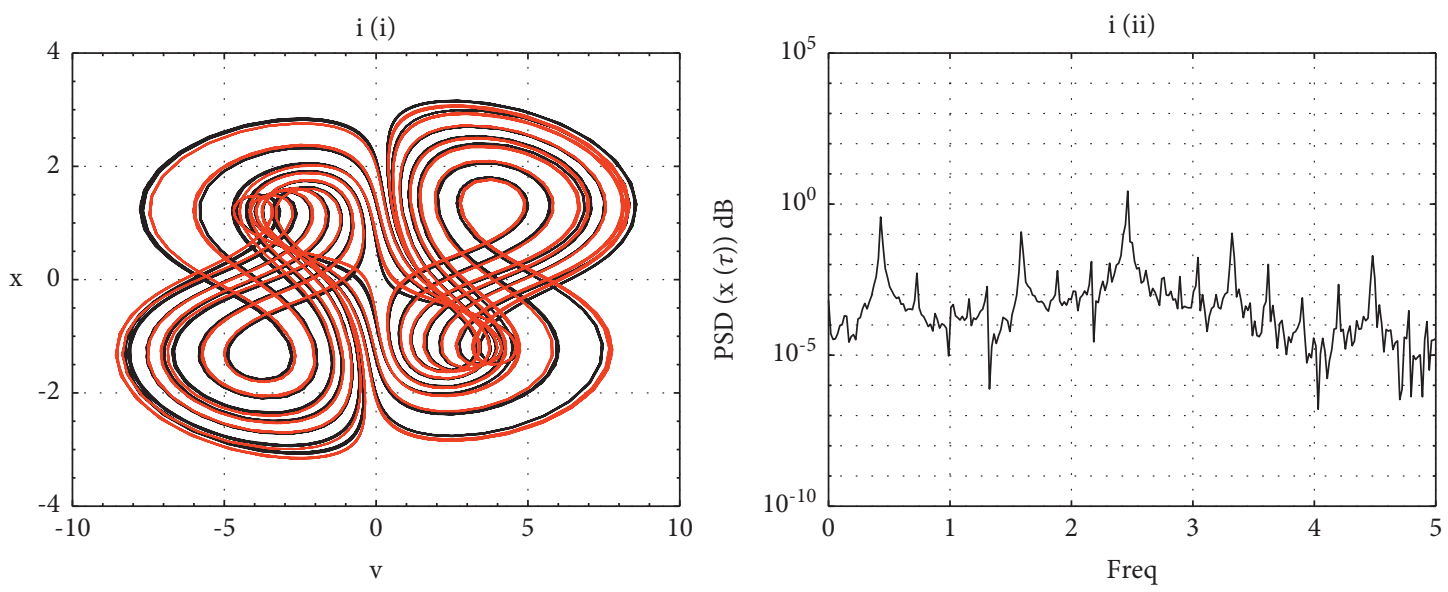

(i)
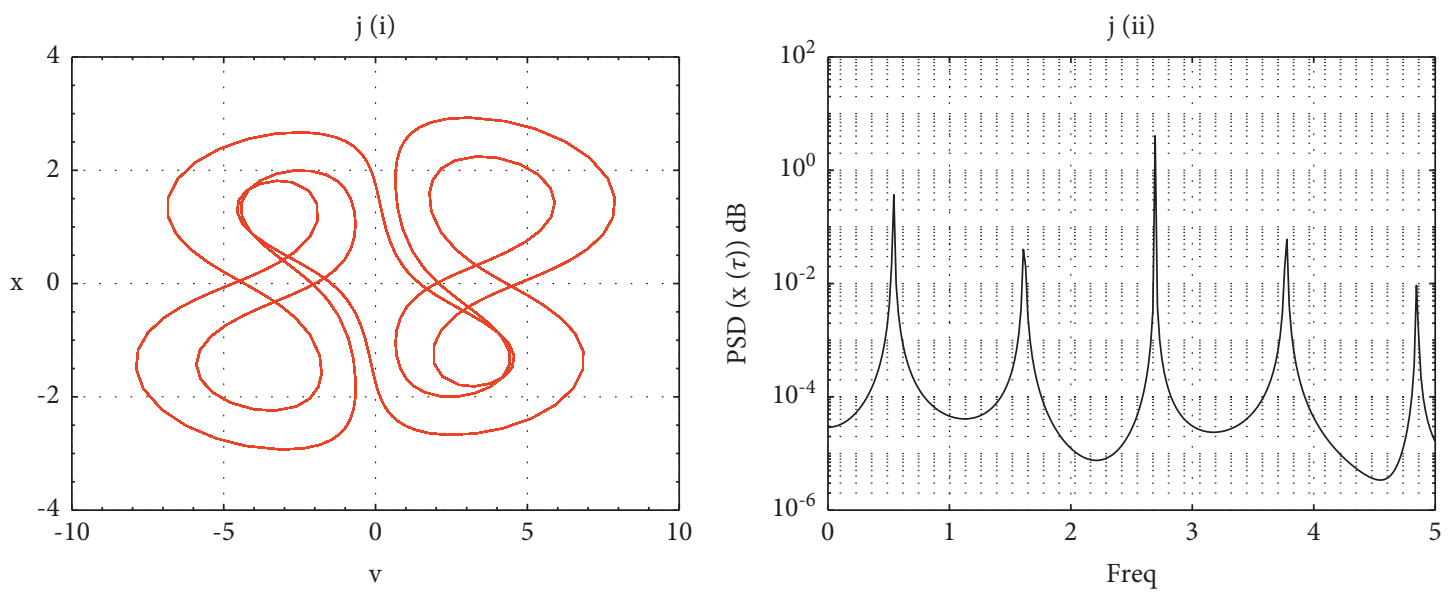

(j)
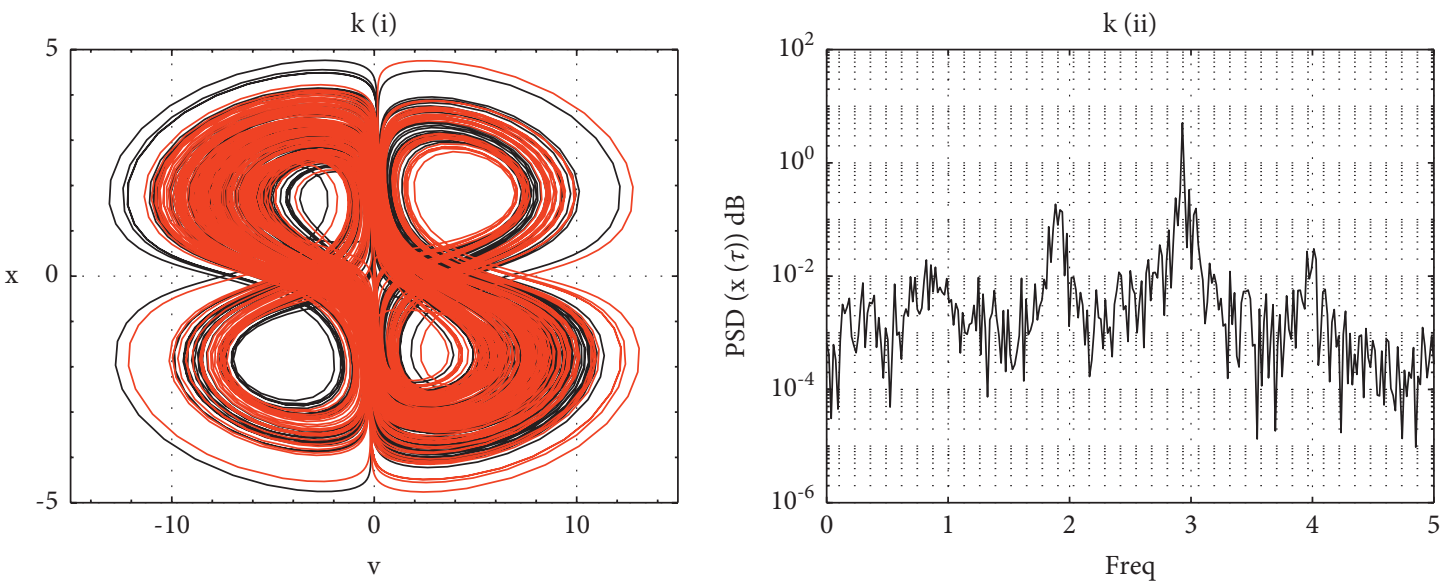

$(\mathrm{k})$

FIGURE 5: Phased portrait revealing the transition to chaos in column (i) with their corresponding power density spectra in column (ii): (a) the pair of chaotic attractors with $\alpha=0.01$; (b) symmetric attractor period-8 with $\alpha=0.1$; (c) symmetric attractor period-2 with $\alpha=0.2$; (d) symmetric attractor period-15 with $\alpha=0.3$; (e) two-wing symmetric chaotic attractor with $\alpha=0.4$; (f) four-wing symmetric chaotic attractor with $\alpha=0.5$; (g) two-wing symmetric period-20 attractor with $\alpha=0.9$; (h) two-wing symmetric full chaotic attractor with $\alpha=1$; (i) four-wing period-9 attractor with $\alpha=1.6$; (j) four-wing period-2 attractor with $\alpha=1.9$; (k) four-wing merge chaotic attractor with $\alpha=3.2$. Initial conditions: $(1,1,1,0.1, \pm 1)$. 


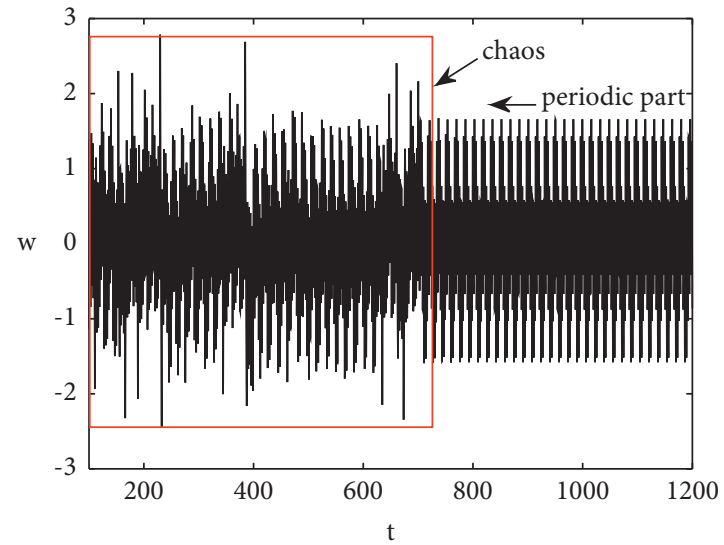

(a)

b (i)

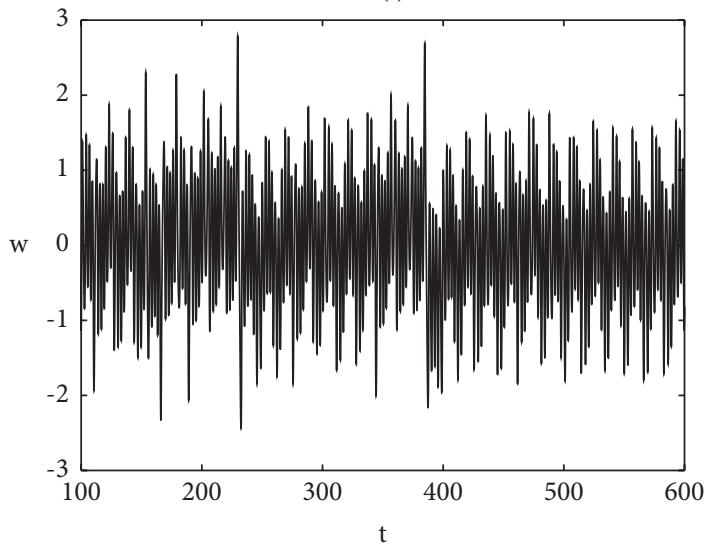

c (i)

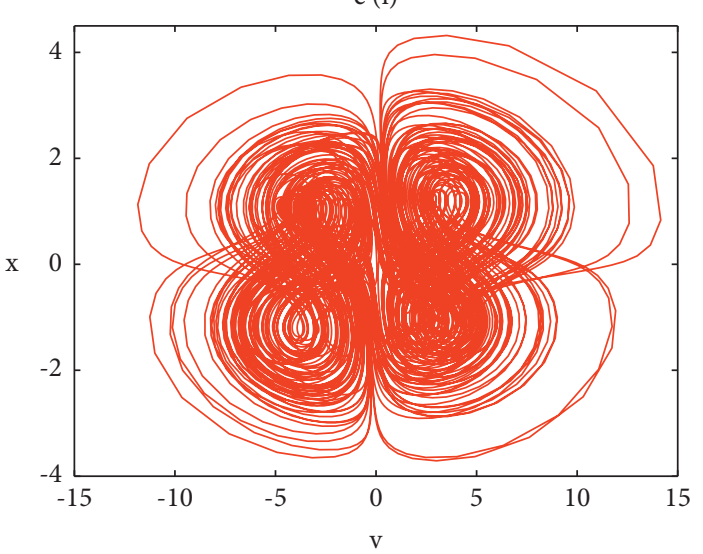

b (ii)

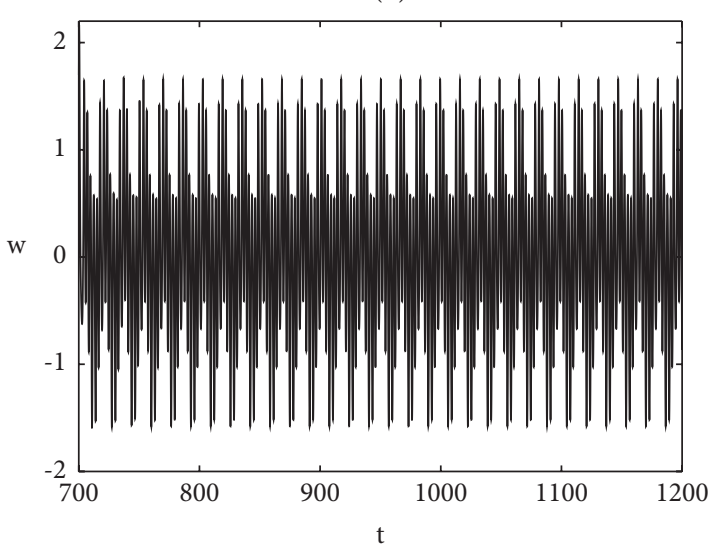

(b)

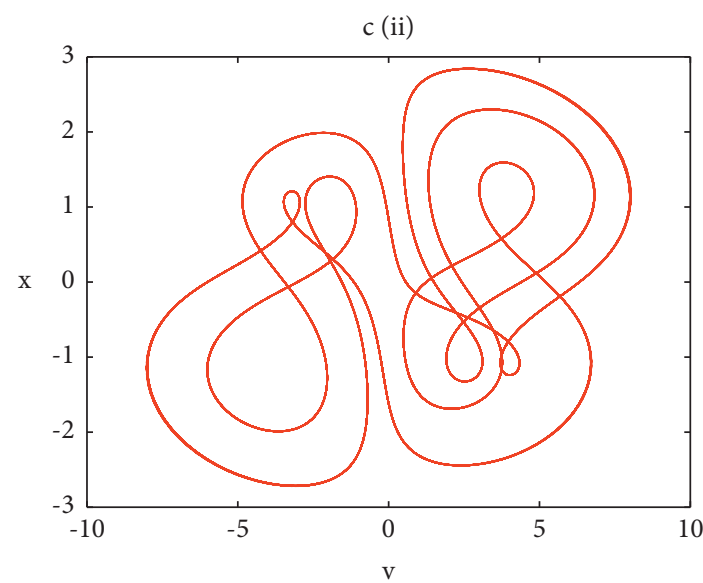

(c)

Figure 6: Continued. 

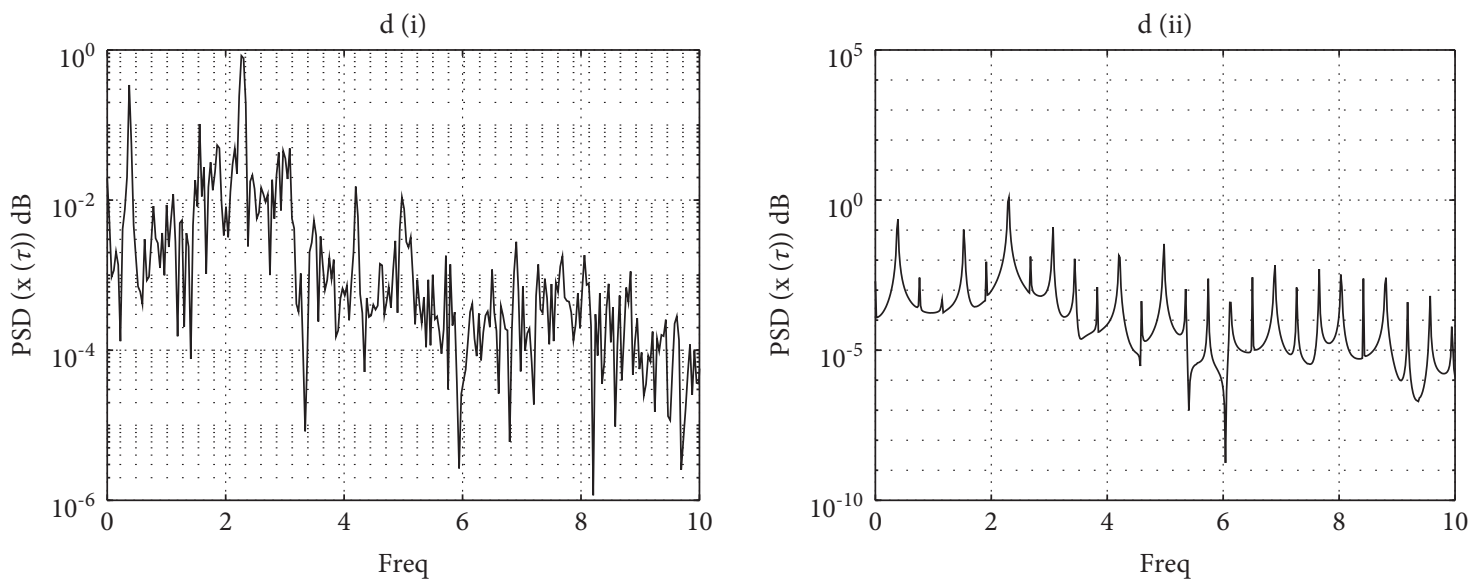

(d)

Figure 6: (a) Time trace of the state variable $x$ of the system while time elapsed. (b) Division of the interval of monotone dynamics: b(i) chaotic; $\mathrm{b}$ (ii) periodic. (c) Corresponding chaotic phase portrait: during time $t<600 \mathrm{~s} \mathrm{c}$ (i) and c(ii) periodic phase portrait during regular motion $t>600 \mathrm{~s}$ (system parameters: $a=0.05, \quad b=0.03 c=6, d=3, e=2, f=1, g=1, k=2, \alpha=1.3, \delta=1 ;$ initial state: $\left.\left(x_{0} ; y_{0} ; z_{0} ; w_{0} ; v_{0}\right)=(1 ; 1 ; 1 ; 0.1 ; 1)\right)$.
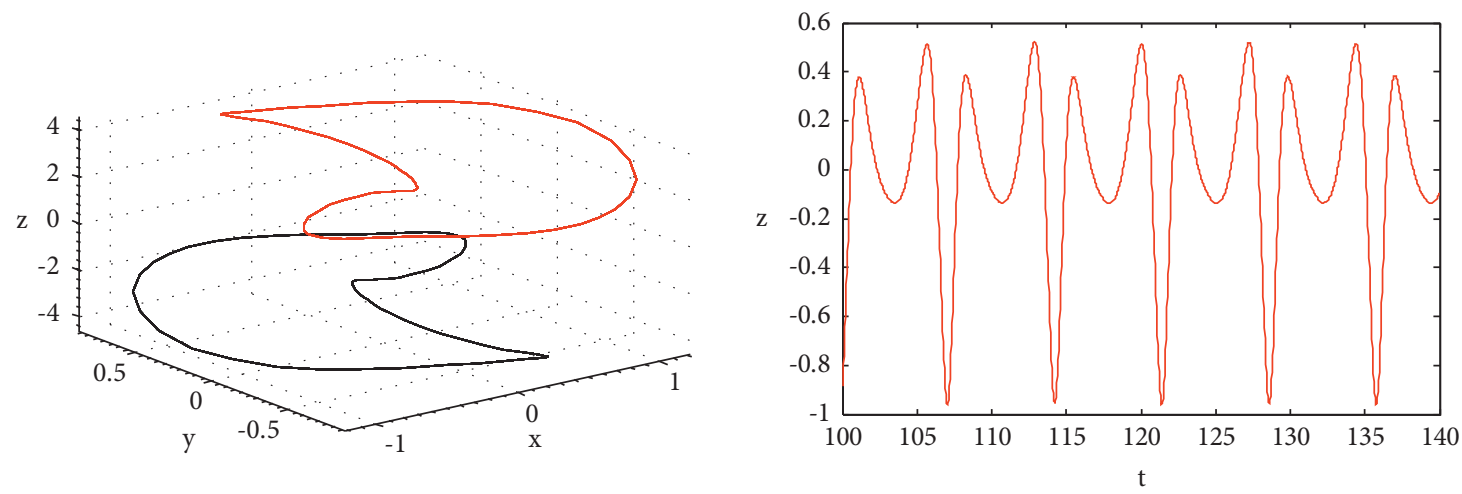

(a)
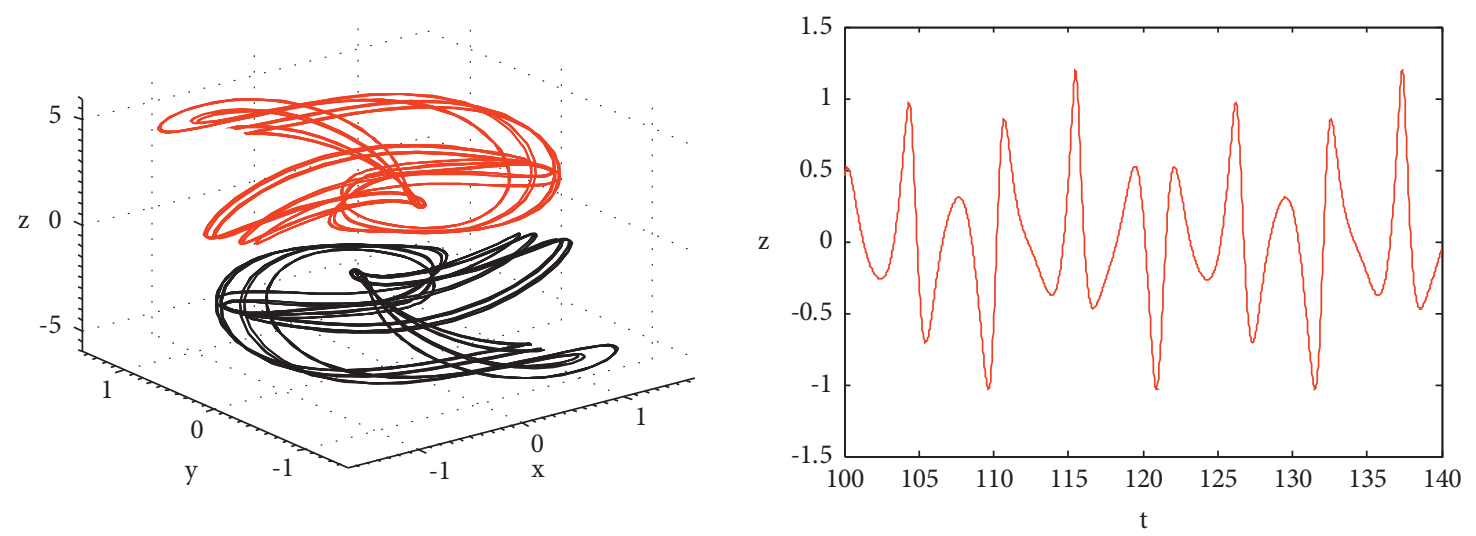

(b)

Figure 7: Continued. 

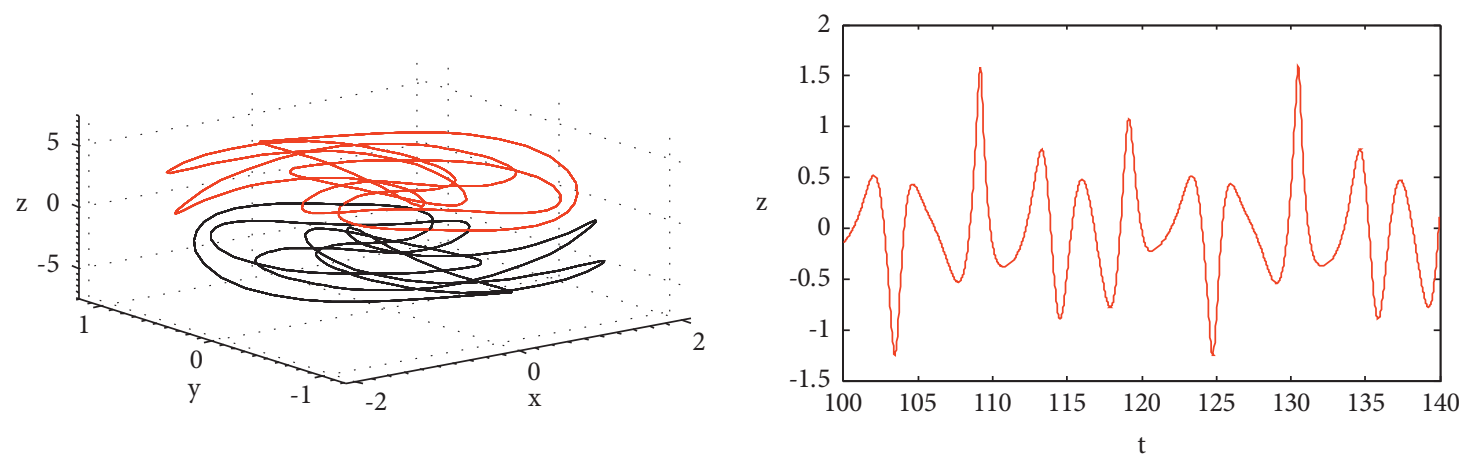

(c)
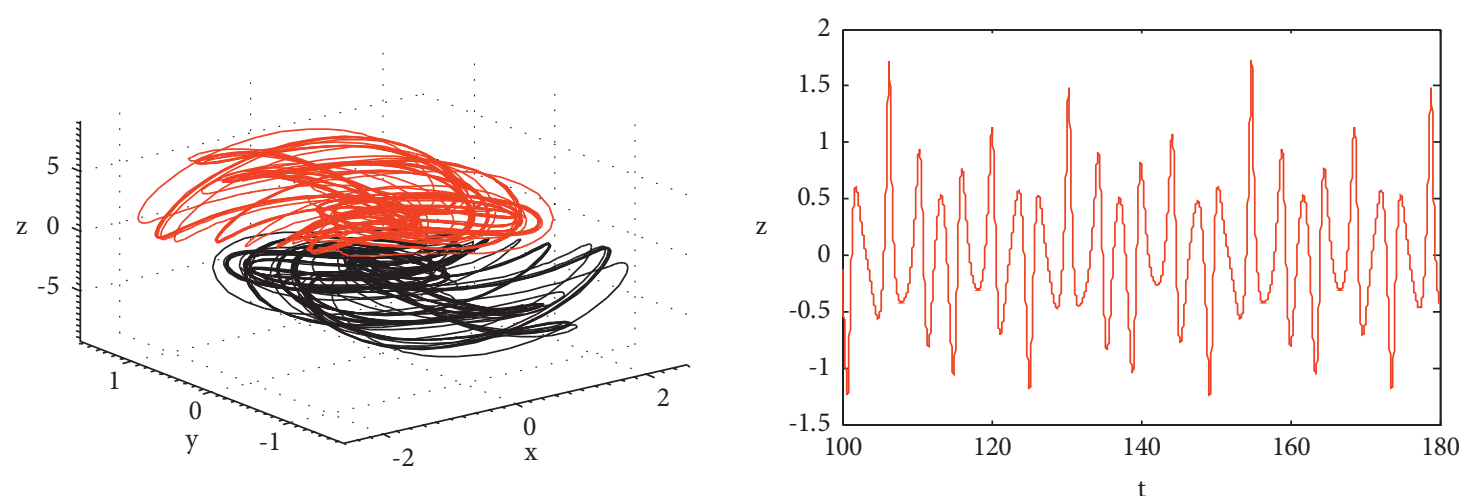

(d)
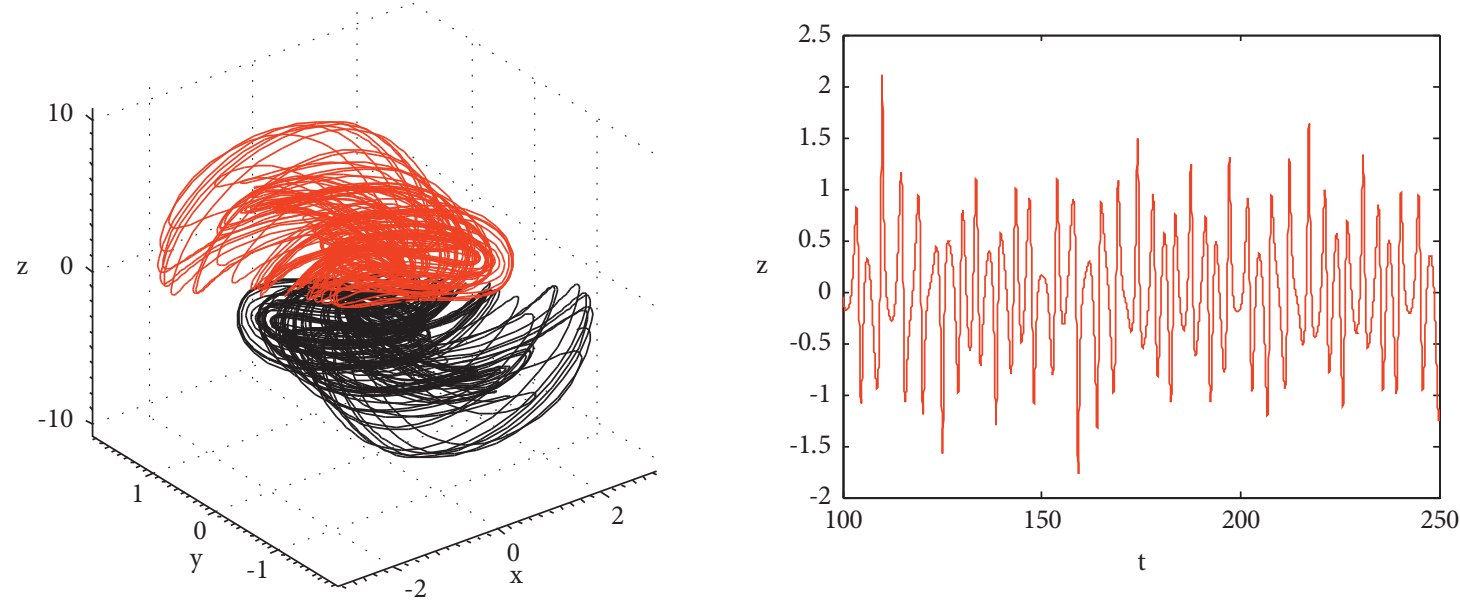

(e)

Figure 7: Continued. 

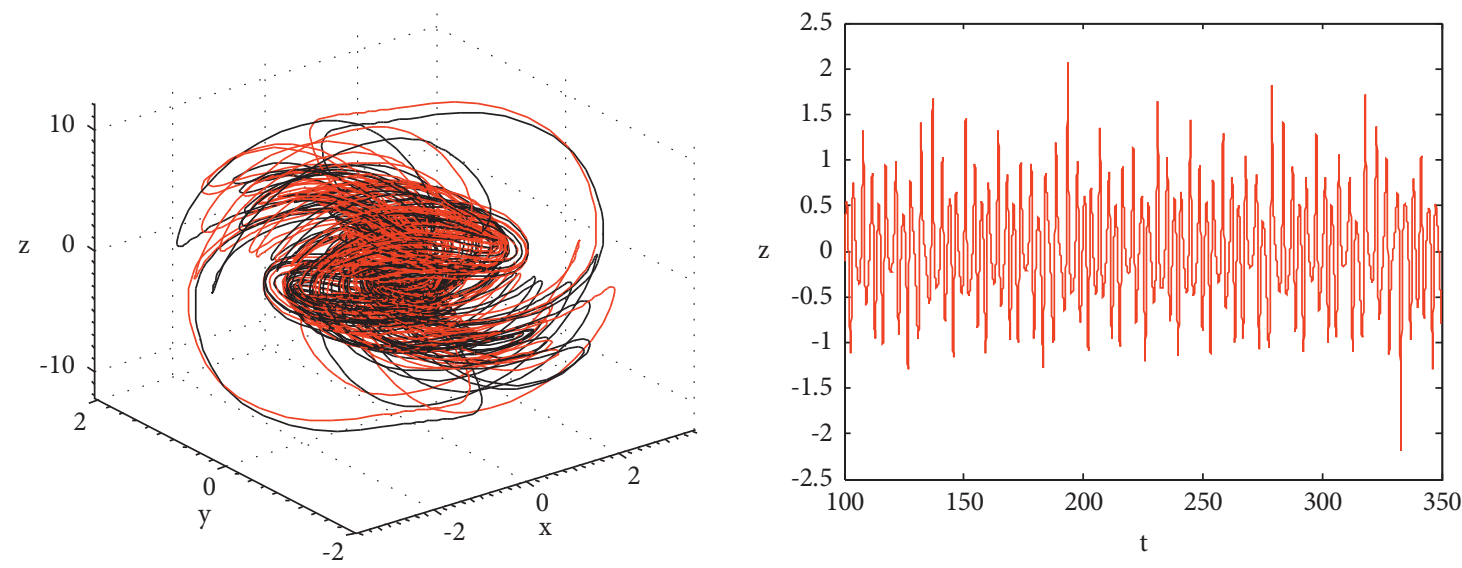

(f)

FigURE $7: 3 \mathrm{D}$ projection of the attractors of system $(3 \mathrm{a})-(3 \mathrm{~d})$ in the $(x, y, z)$ plane (i) and the corresponding time traces (ii) illustrating crisis transitions: the transition from $(\mathrm{e}) \Rightarrow(\mathrm{f})$ indicates the interior crisis behavior while $\alpha$ is varied. Initial conditions $(1 ; 1 ; 1 ; 1 ; 0.01)$ : (a) period-2 for $\alpha=0.2$, (b) period- 6 for $\alpha=0.3$, (c) period- 5 for $\alpha=0.35$, (d) symmetric chaotic attractors for $\alpha=0.4$, (e) $\alpha=0.42$, and (f) chaotic single band for $\alpha=0.43$.

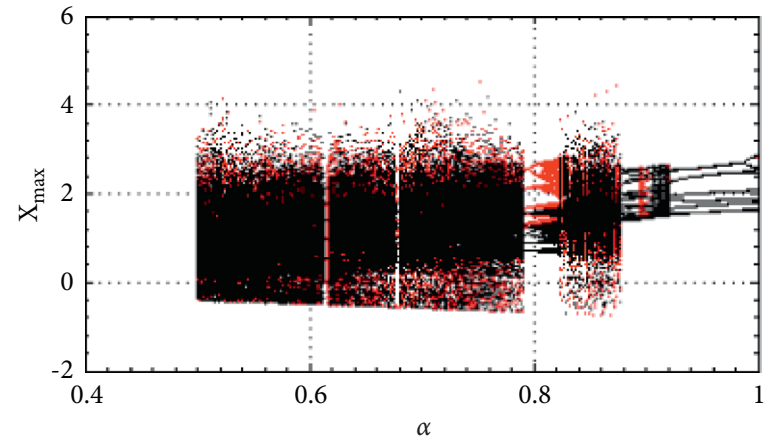

(a)

(b1)

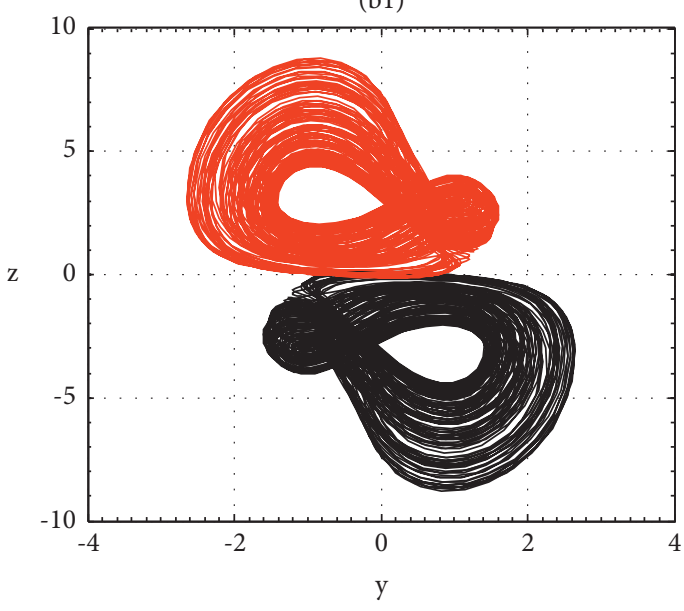

(b2)

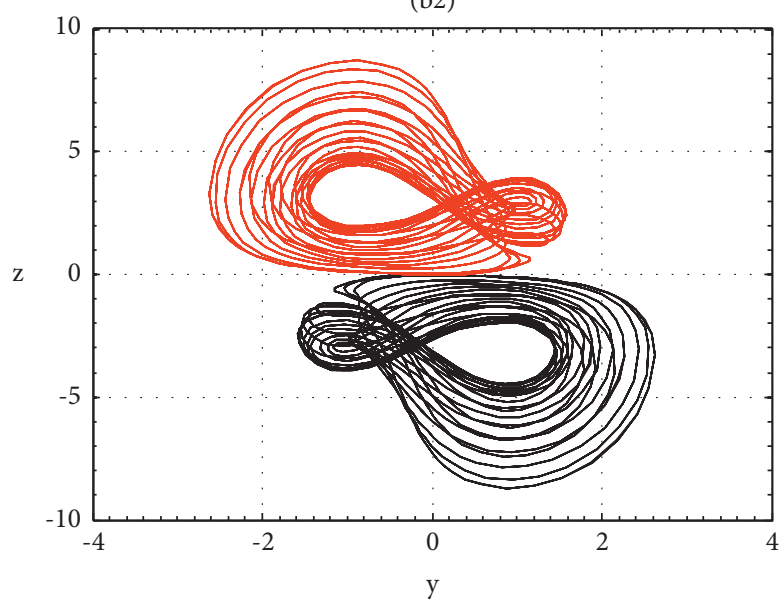

(b)

FIGURE 8: Zoom in the bifurcation diagram (a) and the corresponding discovery of coexistence of 4 attractors $(2$ symmetric chaotic and 2 symmetric periodic attractors) for the same set of parameters $\alpha=0.9, a=0.05, b=0.03, c=6, d=3, e=2, f=1, g=1, k=2, \alpha=1.3, \delta=1$. Initial conditions are $\left(x_{0} ; y_{0} ; z_{0} ; w_{0} ; v_{0}\right)=( \pm 1 ; \pm 1 ; \pm 1 ; \pm 0.1 ; \pm 1)$. 


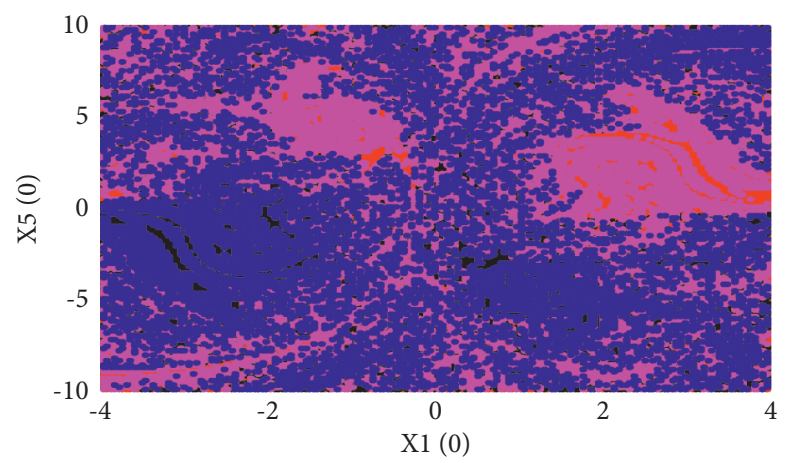

FIGURE 9: Basin of attraction plotted in the plane $\left(x_{1}(0), x_{5}(0)\right)$ showing the initial conditions that lead to each coexisting steady state: the orange area is for positive chaotic attractor while the blue area is for negative ones. The black and red zones are for initial conditions that led to periodic attractors. System parameters are $a=0.05, b=0.03, c=6, d=3, e=2, f=1, g=1, k=2, \alpha=1.3, \delta=1$. The other initial conditions are $X_{2}(0)=1, X_{3}(0)=1, X_{4}(0)=1$ (color version online).

TABLE 2: Lyapunov exponents and dynamics of system (2a)-(2e) with parameter alpha varying.

\begin{tabular}{lccccccc}
\hline$\alpha$ & $\mathrm{LE}_{1}$ & $\mathrm{LE}_{2}$ & $\mathrm{LE}_{3}$ & $\mathrm{LE}_{4}$ & $\mathrm{LE}_{5}$ & Dynamics & Figure \\
\hline 0.525 & 0.119 & 0.00107 & -0.156 & -1.368 & -1.5 & Hyperchaos \\
0.69 & 0.107 & 0.001 & -0.105 & -1.462 & -1.612 & Hyperchaos \\
\hline
\end{tabular}

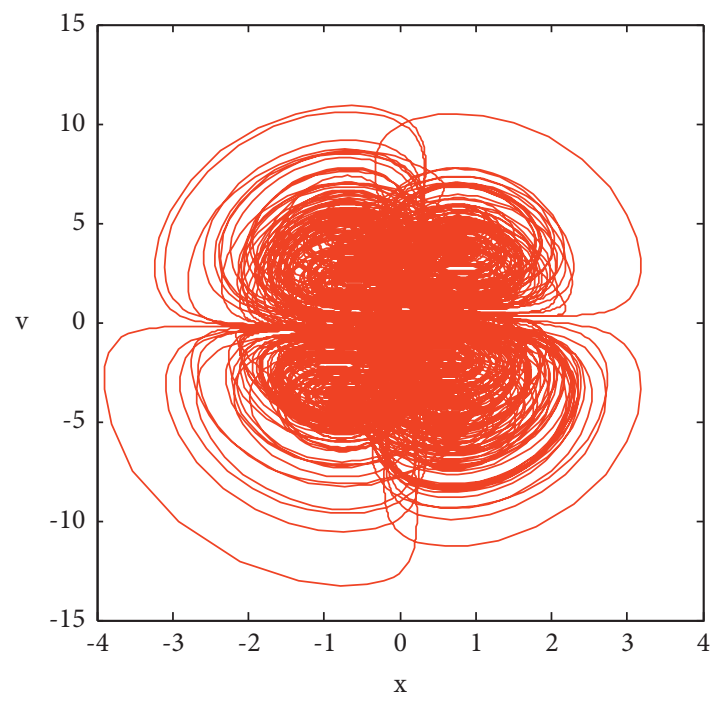

(a)

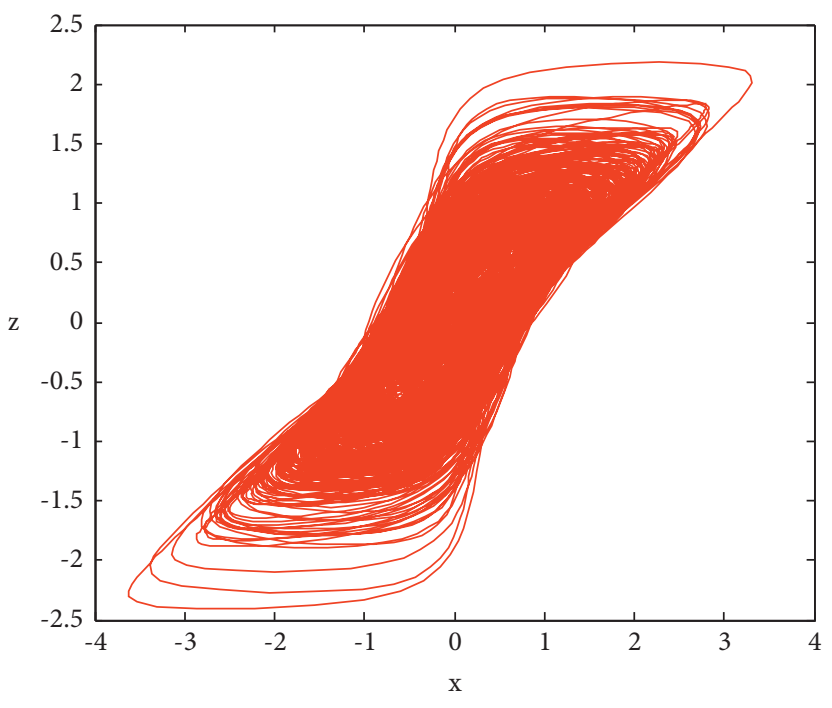

(b)

Figure 10: Phase portraits of the four-scroll hyperchaotic attractor for (a) $\alpha=0.525\left(\operatorname{LE}_{1}=0.119381\right.$ and $\left.\mathrm{LE}_{2}=0.00107263\right)$ and (b) $\alpha=0.69\left(\mathrm{LE}_{1}=0.107339\right.$ and $\left.\mathrm{LE}_{2}=0.001000881\right)$ initial conditions $(x 0 ; y 0 ; z 0 ; w 0 ; v 0)=(1 ; 1 ; 1 ; 0.1 ; 1)$.

3.3.3. Offset Boosting. The flexibility of the Wien Bridge oscillator can be used as a chaotic encoding circuit by means of varying parameters. For this purpose, we exploit the rescaling factor $k$ to illustrate the displaced attractor on the $w$ axis as follows: $w \longrightarrow w+\mu$. System (2a)-(2e) is written as follows:

$$
\begin{aligned}
& \frac{\mathrm{d} x}{\mathrm{~d} t}=\left[c-e\left(a+3 b v^{2}\right)\right] x-d y-e g \sinh (x-z), \\
& \frac{\mathrm{d} y}{\mathrm{~d} t}=c x-d y, \\
& \frac{\mathrm{d} z}{\mathrm{~d} t}=e g \sinh (x-z)-(w+\mu),
\end{aligned}
$$

$$
\begin{aligned}
& \frac{\mathrm{d} w}{\mathrm{~d} t}=k z, \\
& \frac{\mathrm{d} v}{\mathrm{~d} t}=-x-e f\left(-\alpha+\delta x^{2}\right) v .
\end{aligned}
$$

In Figure 11, the attractor moved in the $z$-axis in accordance with the offset variable $\mu$.

3.3.4. Amplitude Control. In this section, we show that the amplitude of the attractor of the oscillator can be varied by means of the scaling factor $m$. In Figure 12, one can see that the dimension attractor can be shrunk or expanded in accordance with the amplitude control factor $m$. 


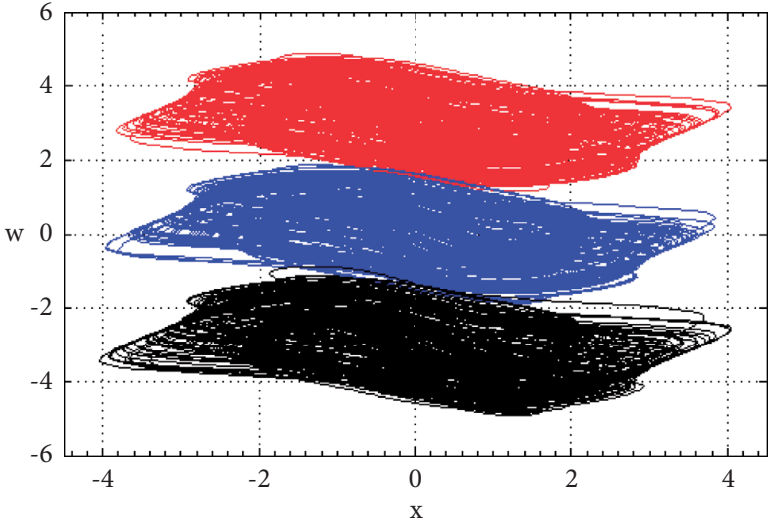

(a)

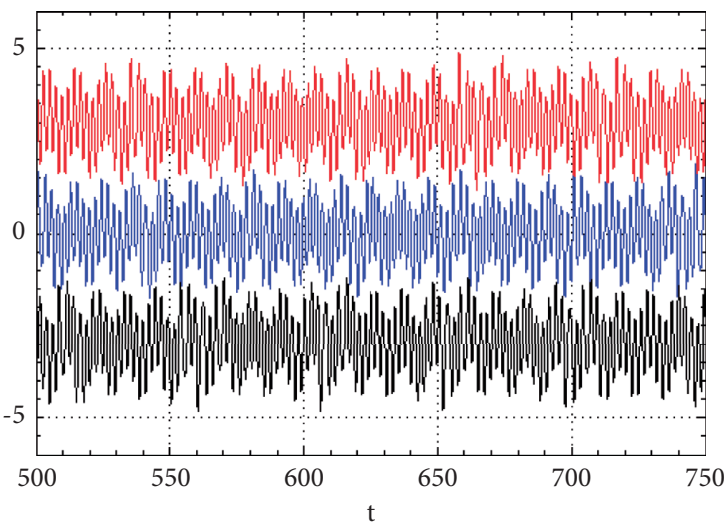

(b)

FIgURE 11: Offset boosting dynamics in $\mathrm{w}$ axis (i.e., $w=w+\mu)$ : (a) phase portraits in $(w, x)$ plane and (b) the time series of $w(t)$ for discrete values of the boosted parameter $\mu: \mu=-3$ red; $\mu=0$ blue; $\mu=+3$ green. Initial conditions: $x(0)=1, y(0)=1, z(0)=1, w(0)=1, v(0)=0.01$. The rest of the system (2a)-(2e) parameters are in Figure 2.

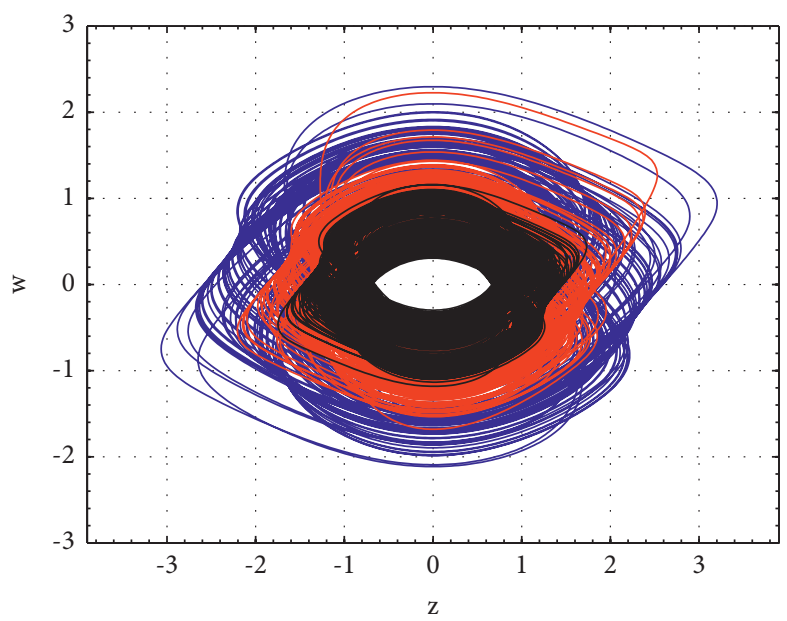

Figure 12: Total amplitude control dynamics by substitution of state variables $x \longrightarrow x / m, y$ by $y / m, z$ by $z / m, w$ by $w / m$, and $v$ by $v / m$ shown by means of (a) phase portraits in $(z, w)$ plane and (b) $(x, z)$ plane for discrete values of the amplitude parameter $m: m=0.5$ red; $m=0.2$ blue; $m=30$ black. The rest of the system (3a)-(3d) parameters are in Figure 2; $t$ is the dimensionless time.

As we can see, the striking amplitude behavior can be exploited in engineering instrumentation.

3.3.5. Bursting Oscillations. By selecting some discrete values of the parameter $\alpha=0.01$, the time series and phase portraits of $y$ versus $x$ and $z$ versus $y$ are displayed in Figures 13(iii) and (iv) illustrating the periodic bursting oscillations. According to these figures, one can observe that sometimes, the system is fast while other times, it is slow in regular space by a constant period, $T=50 \mathrm{~s}$.

\section{Electronic Implementation of a Memristive Wien Bridge Oscillator}

In this section, the objective of the study is to verify some interesting behaviors found during the numerical simulation of the model of the Wien bridge oscillator with real antiparallel diodes.
It is important to note that recently, numerous advantages of digital components like FPGA, FPAA, DSP, and SOC have made them suitable for the implementation of chaotic systems just to name a few compared to the analog electronics component. Among them, the microcontrollers offer more flexibility for setting control parameters and initial conditions accurately, reducing the system to a portable source code, and realizing complicated mathematical operations or algorithms without needing special tools. These features increase the number of realizable chaotic systems and simplify the implementation process [64-67].

For this purpose, the experimental setup is drawn in Figure 14 .

The experimental setup is composed of an Arduino MEGA board powered by a $9 \mathrm{~V}$ DC battery. The computer is connected to the USB port of the Arduino card built with an ATMEGA2560 microprocessor. It is connected to the computer to display data from the Arduino MEGA interface. 

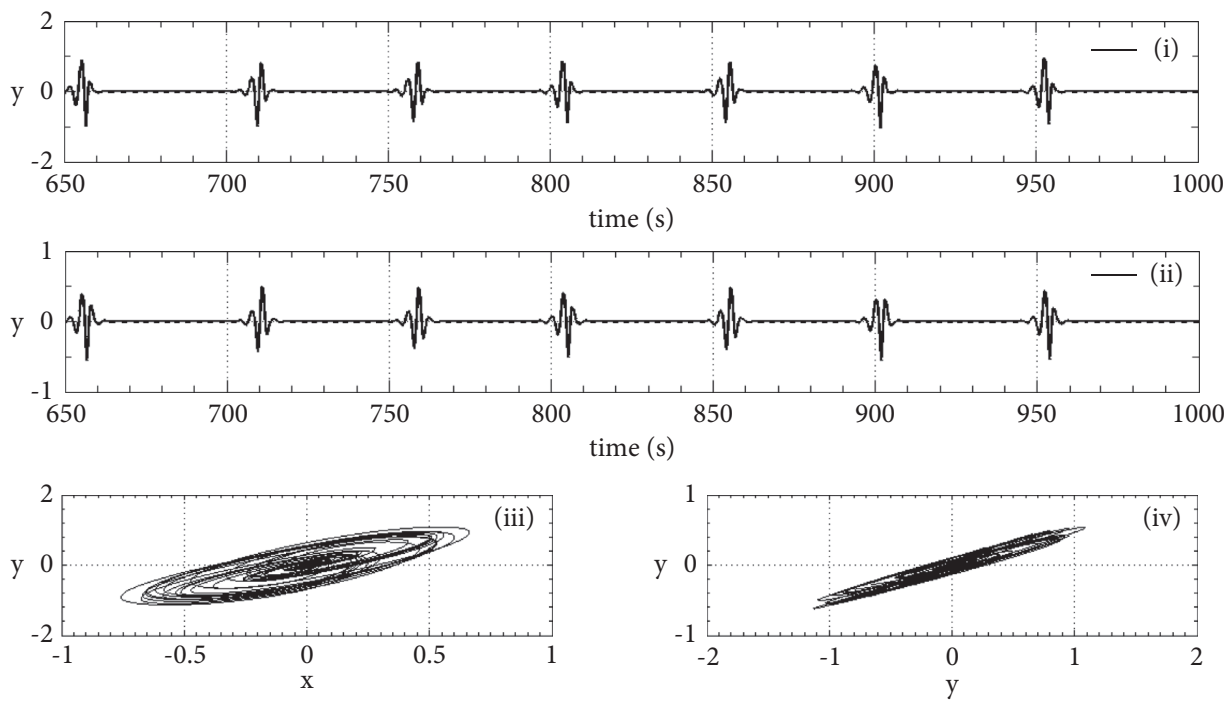

FIgure 13: Periodic bursting oscillations of the Wien Bridge Oscillator: (i) + (ii) time traces of $y$ and $z$; (iii) + (iv) 2D phase portraits. Parameters of the system are $\alpha=0.01$; the other ones are in Figure 8 .

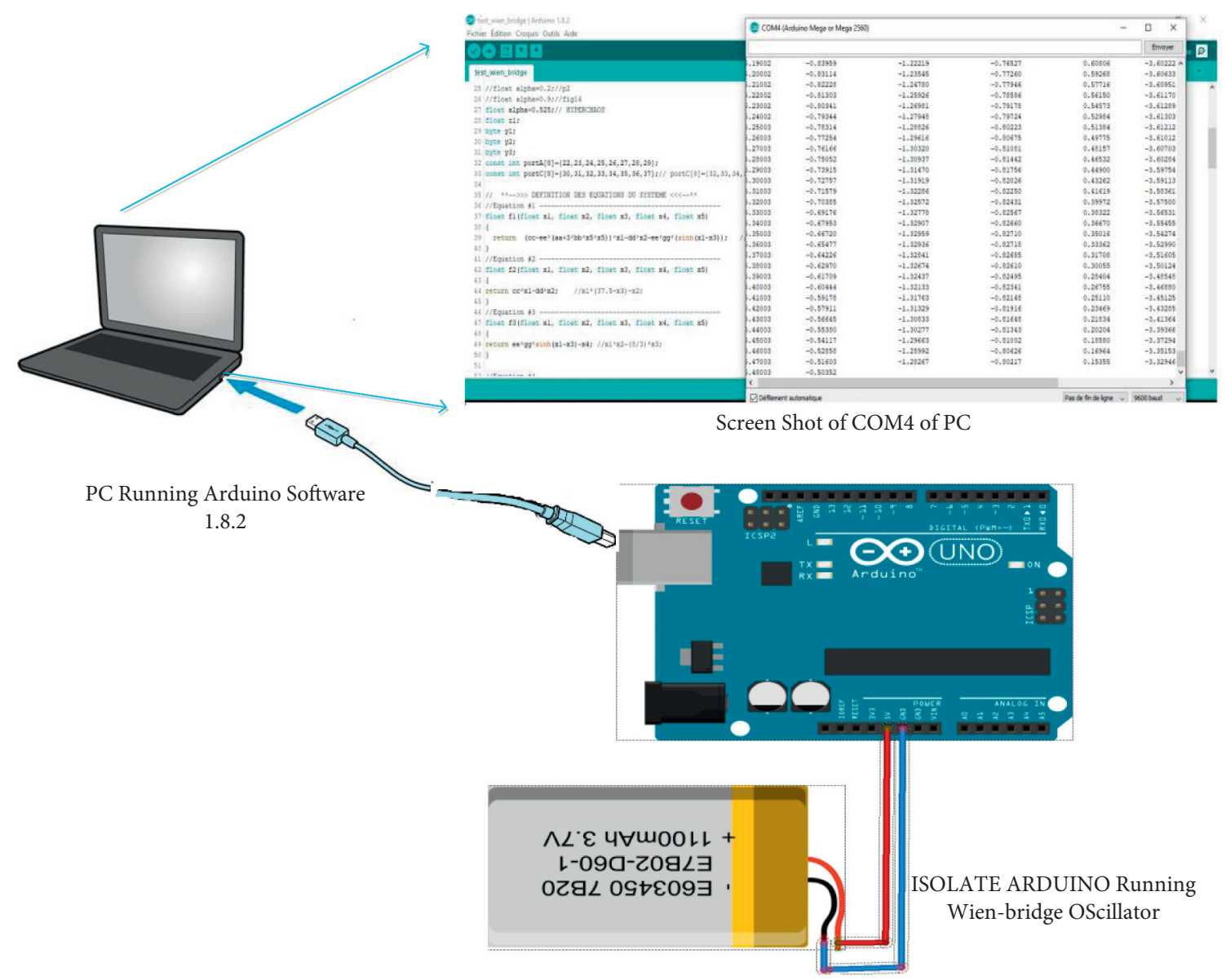

FIgURE 14: Microcontroller experiment workbench of the Wien bridge oscillator.

The memristive Wien bridge oscillator described by system (1a)-(1e) is transformed in numerical form by means of the $4^{\text {th }}$ order Runge-Kutta numerical method with a tiny step time of $0.005 \mathrm{~s}$, typed in the Arduino software, and downloaded in the Arduino MEGA. The resulting real state variables $x, y, z, w, v$ are sent to the serial monitor of the software from the analog signal. Figures 15 and 16 show the phase planes obtained from the microcontroller. 


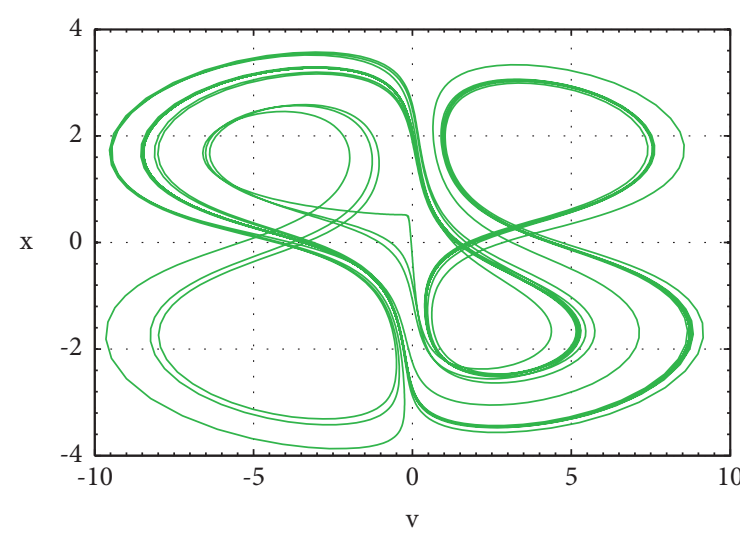

(a)

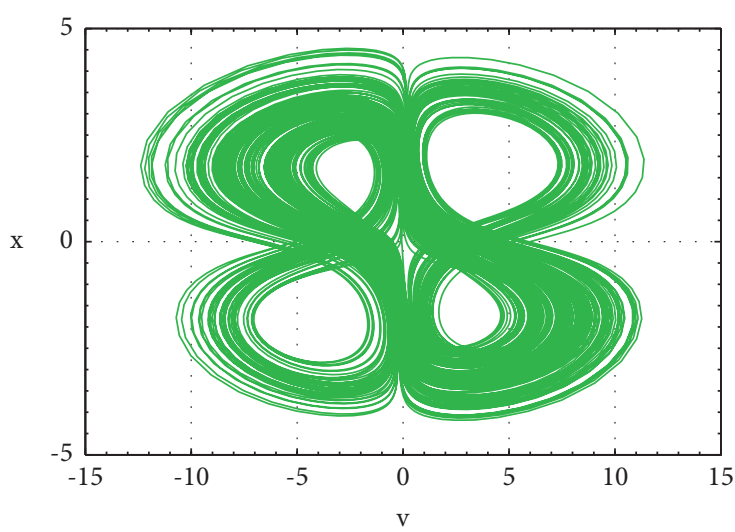

(b)

FIGURE 15: Four-wing phased portraits of the Arduino-based implementation of the Wien bridge oscillator confirming the intermittency chaotic scenario for external input $A_{0}$. (a) $A_{0}=2.95$ period-2 and (b) $A_{0}=3.2$ chaotic; this figure reassembles Figure 8(m), (n).

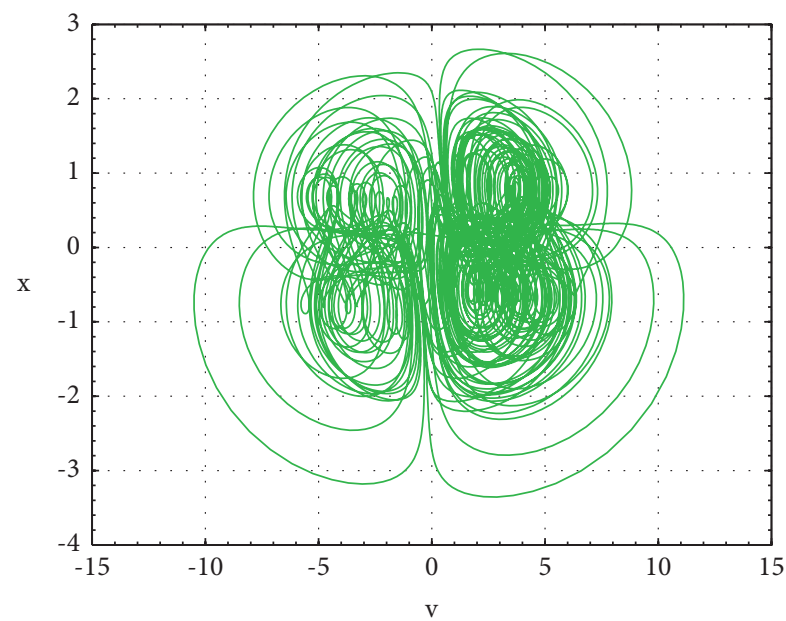

FIGURE 16: Experimental phase portraits of the four-scroll hyperchaotic attractor: (a) $\alpha=0.525$ starting points are $(1 ; 1 ; 1 ; 3.1 ; 1)$.

As the reader can see, the experimental design produces on the screen of the computer data that are captured and plotted in MATLAB software for comparison purposes and similar results are recovered.

\subsection{Intermittency Route to Chaos with Symmetry Crisis Verification}

4.2. Hyperchaotic Behavior. As we can see, Figure 15 reproduces the intermittency scenario and Figure 16 the hyperchaotic dynamic of the real component.

\section{Conclusion}

In this contribution, a new Wien bridge oscillator was introduced and analyzed. The investigations show that some additional behavior found apart from those already revealed in this oscillator was discovered, namely, intermittency route to chaos, transient chaos, hyperchaos with offset boosting, partial amplitude control, and bursting oscillations. The route to chaos is intermittent, transient chaos with some multistability characterized by the coexistence of up to 4 attractors for the same set of parameters. A basin of attraction is the plot to highlight this scenario. Experiment results based on an isolated Arduino card-built ATMEGA2560 processor producing a digital output of each state variable of the Wien bridge oscillator are consistent with theoretical and numerical predictions. With the research on fracmemristor $[68,69]$ increasing with high interest, the outlook of this paper is to propose the fractional version of the circuit in Figure 1 with fracmemristor to increase the complexity of the nonlinear Wien bridge oscillator because it can increase the number of disconnected attractors of the Wien bridge. The dimension of the system under scrutinizing is $5 \mathrm{D}$ greater than the ones in the literature, but the presence of 4-wing hyperchaotic attractors is an important metric for the system to be used as an image encryption oscillator embedded on an Arduino microcontroller. Therefore, we are planning in the nearest future to experiment with this fascinating application.

\section{Data Availability}

No data were used to support this study.

\section{Conflicts of Interest}

The authors declare they have no conflicts of interest.

\section{Acknowledgments}

The authors sincerely thank Serdar ÇİÇEK of Vocational School of Hacıbektaş, Nevşehir Hacı Bektaş Veli University, 50800 Hacıbektaş, Nevşehir, Turkey, for fruitful discussion on arduino based implementation. Karthikeyan Rajagopal was partially supported by the Institute of 
Research and Development, Defence University, Ethiopia (Grant no. CND/DEC/2018-10).

\section{References}

[1] W.-K. Ling, Control of Chaos in Nonlinear Circuits and Systems, Vol. 64, World Scientific, Singapore, 2009.

[2] I. Nagy, "Nonlinear phenomena in power electronics," Automatika-Zagreb, vol. 42, no. 3/4, pp. 117-132, 2001.

[3] T. L. Carroll and L. M. Pecora, Nonlinear Dynamics in Circuits, World Scientific, Singapore, 1995.

[4] G. Chen and T. Ueta, "Chaos in circuits and systems," vol. 11Singapore, World Scientific, 2002.

[5] M. E. Sahin, Z. G. C. Taskiran, H. Guler, and S. E. Hamamci, "Application and modeling of a novel 4D memristive chaotic system for communication systems," Circuits, Systems, and Signal Processing, vol. 39, no. 7, pp. 3320-3349, 2020.

[6] B. Karakaya, A. Gülten, and M. Frasca, "A true random bit generator based on a memristive chaotic circuit: analysis, design and FPGA implementation," Chaos, Solitons \& Fractals, vol. 119, pp. 143-149, 2019.

[7] M. Wang, Y. Deng, X. Liao, Z. Li, M. Ma, and Y. Zeng, "Dynamics and circuit implementation of a four-wing memristive chaotic system with attractor rotation," International Journal of Non-linear Mechanics, vol. 111, pp. 149-159, 2019.

[8] M. E. Sahin, Z. G. Cam Taskiran, H. Guler, and S. E. Hamamci, "Simulation and implementation of memristive chaotic system and its application for communication systems," Sensors and Actuators A: Physical, vol. 290, pp. 107-118, 2019.

[9] V. T. Pham, S. Jafari, S. Vaidyanathan, C. Volos, and X. Wang, "A novel memristive neural network with hidden attractors and its circuitry implementation," Science China Technological Sciences, vol. 59, no. 3, pp. 358-363, 2016.

[10] J. Kengne, Z. Njitacke Tabekoueng, V. Kamdoum Tamba, and A. Nguomkam Negou, "Periodicity, chaos, and multiple attractors in a memristor-based Shinriki's circuit," Chaos: An Interdisciplinary Journal of Nonlinear Science, vol. 25, no. 10, Article ID 103126, 2015.

[11] Z. T. Njitacke, J. kengne, H. B. Fotsin, A. N. Negou, and D. Tchiotsop, "Coexistence of multiple attractors and crisis route to chaos in a novel memristive diode bidge-based Jerk circuit," Chaos, Solitons \& Fractals, vol. 91, pp. 180-197, 2016.

[12] Q. Xu, Q. L. Zhang, H. Qian, H. G. Wu, and B. C. Bao, "Crisisinduced coexisting multiple attractors in a second-order nonautonomous memristive diode bridge-based circuit," International Journal of Circuit Theory and Applications, vol. 46, no. 10, pp. 1917-1927, 2018.

[13] B. C. Bao, H. Bao, N. Wang, M. Chen, and Q. Xu, "Hidden extreme multistability in memristive hyperchaotic system," Chaos, Solitons \& Fractals, vol. 94, pp. 102-111, 2017.

[14] J. R. M. Pone, F. Momo, G. Honore Kom, I. Ornela Lowe Kombou, S. Takougang Kingni, and A. Tiedeu, "Extreme multistability, hidden chaotic attractors and amplitude controls in an absolute memristor Van der Pol-duffing circuit: dynamical analysis and electronic implementation." in In Mem-Elements for Neuromorphic Circuits with Artificial Intelligence Applications, pp. 165-181, Academic Press, Cambridge, MA, USA, 2021.

[15] B. C. Bao, P. Y. Wu, H. Bao, H. G. Wu, X. Zhang, and M. Chen, "Symmetric periodic bursting behavior and bifurcation mechanism in a third-order memristive diode bridge- based oscillator," Chaos, Solitons \& Fractals, vol. 109, pp. 146-153, 2018.

[16] X. Ye, J. Mou, C. Luo, and Z. Wang, "Dynamics analysis of Wien-bridge hyperchaotic memristive circuit system," Nonlinear Dynamics, vol. 92, no. 3, pp. 923-933, 2018.

[17] Y. Song, F. Yuan, and Y. Li, "Coexisting attractors and multistability in a simple memristive Wien-bridge chaotic circuit," Entropy, vol. 21, no. 7, p. 678, 2019.

[18] A. Jhangeer, M. Muddassar, Z. Ur Rehman, J. Awrejcewicz, and M. B. Riaz, "Multistability and dynamic behavior of nonlinear wave solutions for analytical kink periodic and quasiperiodic wave structures in plasma physics," Results in Physics, vol. 29, Article ID 104735, 2021.

[19] S. He, H. Natiq, and S. Mukherjee, "Multistability and chaos in a noise-induced blood flow," The European Physical JournalSpecial Topics, vol. 230, no. 5, pp. 1525-1533, 2021.

[20] M. Morozov, "Adsorption inhibition by swollen micelles may cause multistability in active droplets," Soft Matter, vol. 16, no. 24, pp. 5624-5632, 2020.

[21] A. Sambas, S. Vaidyanathan, E. Tlelo-Cuautle et al., "A 3-D multi-stable system with a peanut-shaped equilibrium curve: circuit design, FPGA realization, and an application to image encryption," IEEE Access, vol. 8, pp. 137116-137132, 2020.

[22] A. Sambas, S. Vaidyanathan, S. Zhang, Y. Zeng, M. A. Mohamed, and M. Mamat, "A new double-wing chaotic system with coexisting attractors and line equilibrium: bifurcation analysis and electronic circuit simulation," IEEE Access, vol. 7, pp. 115454-115462, 2019.

[23] A. Sambas, fnm Vaidyanathan, fnm Tlelo-Cuautle et al., "A novel chaotic system with two circles of equilibrium points: multistability, electronic circuit and FPGA realization," Electronics, vol. 8, no. 11, p. 1211, 2019.

[24] R. C. Hilborn, Chaos and Nonlinear Dynamics: An Introduction for Scientists and Engineers, Oxford University Press on Demand, Oxford, UK, 2000.

[25] S. Mondal, S. A. Pawar, and R. I. Sujith, "Synchronous behaviour of two interacting oscillatory systems undergoing quasiperiodic route to chaos," Chaos: An Interdisciplinary Journal of Nonlinear Science, vol. 27, no. 10, Article ID 103119, 2017.

[26] M. Gupta and M. Ruzzene, "Dynamics of quasiperiodic beams," Crystals, vol. 10, no. 12, p. 1144, 2020.

[27] N. Ichinose, "Quasiperiodic-chaotic neural networks and short-term analog memory," International Journal of Bifurcation and Chaos, vol. 31, no. 1, Article ID 2130003, 2021.

[28] X. Wu, H. Wang, and H. Lu, "Modified generalized projective synchronization of a new fractional-order hyperchaotic system and its application to secure communication," Nonlinear Analysis: Real World Applications, vol. 13, no. 3, pp. 14411450, 2012.

[29] A. Ishaq Ahamed and M. Lakshmanan, "Nonsmooth bifurcations, transient hyperchaos and hyperchaotic beats in a memristive Murali-Lakshmanan-Chua circuit," International Journal of Bifurcation and Chaos, vol. 23, no. 6, Article ID 1350098, 2013.

[30] A. S. Hegazi and A. E. Matouk, "Dynamical behaviors and synchronization in the fractional order hyperchaotic Chen system," Applied Mathematics Letters, vol. 24, no. 11, pp. 1938-1944, 2011.

[31] Z. Cao and L. Wang, "A secure video watermarking technique based on hyperchaotic Lorentz system," Multimedia Tools and Applications, vol. 78, no. 18, pp. 26089-26109, 2019.

[32] L. Holden and T. Erneux, "Understanding bursting oscillations as periodic slow passages through bifurcation and limit 
points," Journal of Mathematical Biology, vol. 31, no. 4, pp. 351-365, 1993.

[33] S. T. Kingni, B. Nana, G. S. Mbouna Ngueuteu, P. Woafo, and J. Danckaert, "Bursting oscillations in a 3D system with asymmetrically distributed equilibria: mechanism, electronic implementation and fractional derivation effect," Chaos, Solitons \& Fractals, vol. 71, pp. 29-40, 2015.

[34] S. T. Kingni, L. Keuninckx, P. Woafo, G. V. D. Sande, and J. Danckaert, "Dissipative chaos, Shilnikov chaos and bursting oscillations in a three-dimensional autonomous system: theory and electronic implementation," Nonlinear Dynamics, vol. 73, no. 1, pp. 1111-1123, 2013.

[35] E. M. Izhikevich, "Neural excitability, spiking and bursting," International Journal of Bifurcation and Chaos, vol. 10, no. 6, pp. 1171-1266, 2000.

[36] J. Jerrelind and A. Stensson, "Nonlinear dynamics of parts in engineering systems," Chaos, Solitons \& Fractals, vol. 11, no. 15 , pp. 2413-2428, 2000.

[37] A. Kadivar and K. Niayesh, "Simulation of free burning arcs and arcs inside Cylindrical Tubes initiated by exploding wires," in Proceedings of the 2019 5th International Conference on Electric Power Equipment-Switching Technology (ICEPEST), October 2019.

[38] C. Grebogi, E. Ott, and J. A. Yorke, "Crises, sudden changes in chaotic attractors, and transient chaos," Physica D: Nonlinear Phenomena, vol. 7, no. 1-3, pp. 181-200, 1983.

[39] H. M. Osinga and U. Feudel, "Boundary crisis in quasi periodically forced systems," Physica D: Nonlinear Phenomena, vol. 141, no. 1-2, pp. 54-64, 2000.

[40] L. Zhi-Jun and Z. Yi-Cheng, "A memristor chaotic circuit based on Wien-bridge oscillator," Journal of Electronics and Information Technology, vol. 36, no. 1, pp. 88-93, 2014.

[41] H. Wu, B. Bao, Z. Liu, Q. Xu, and P. Jiang, "Chaotic and periodic bursting phenomena in a memristive Wien-bridge oscillator," Nonlinear Dynamics, vol. 83, no. 1, pp. 893-903, 2016.

[42] J. R. Mboupda Pone, S. Çiçek, S. Takougang Kingni, A. Tiedeu, and M. Kom, "Passive-active integrators chaotic oscillator with anti-parallel diodes: analysis and its chaos-based encryption application to protect electrocardiogram signals," Analog Integrated Circuits and Signal Processing, vol. 103, no. 1, pp. 1-15, 2019.

[43] L. Kamdjeu Kengne, H. T. Kamdem Tagne, A. N. Kengnou Telem, J. R. Mboupda Pone, and J. Kengne, "A broken symmetry approach for the modeling and analysis of antiparallel diodes-based chaotic circuits: a case study," Analog Integrated Circuits and Signal Processing, vol. 104, no. 2, pp. 205-227, 2020.

[44] J. C. Sprott, "A proposed standard for the publication of new chaotic systems," International Journal of Bifurcation and Chaos, vol. 21, no. 9, pp. 2391-2394, 2011.

[45] X. Ye, X. Wang, J. Mou, X. Yan, and Y. Xian, "Characteristic analysis of the fractional-order hyperchaotic memristive circuit based on the Wien bridge oscillator," The European Physical Journal Plus, vol. 133, no. 12, p. 516, 2018.

[46] B. Muthuswamy and L. O. Chua, "Simplest chaotic circuit," International Journal of Bifurcation and Chaos, vol. 20, no. 05, pp. 1567-1580, 2010.

[47] L. Teng, H. C. I. Herbert, X. Wang, and X. Wang, "Chaotic behavior in fractional-order memristor-based simplest chaotic circuit using fourth degree polynomial," Nonlinear Dynamics, vol. 77, no. 1, pp. 231-241, 2014.

[48] W. H. Press et al., Numerical Recipes, Vol. 818, Cambridge University Press, Cambridge, 1986.
[49] W. Marszalek, H. Podhaisky, and J. Sadecki, "Computing twoparameter bifurcation diagrams for oscillating circuits and systems," IEEE Access, vol. 7, pp. 115829-115835, 2019.

[50] A. Wolf, J. B. Swift, H. L. Swinney, and J. A. Vastano, "Determining Lyapunov exponents from a time series," Physica D: Nonlinear Phenomena, vol. 16, no. 3, pp. 285-317, 1985.

[51] N. Berglund, Adiabatic Dynamical Systems and Hysteresis, EPFL, Berglund, Nils, 1998.

[52] R. Benzi, G. Paladin, G. Parisi, and A. Vulpiani, "Characterisation of intermittency in chaotic systems," Journal of Physics A: Mathematical and General, vol. 18, no. 12, pp. 2157-2165, 1985.

[53] T. Tél and Y.-C. Lai, "Chaotic transients in spatially extended systems,” Physics Reports, vol. 460, no. 6, pp. 245-275, 2008.

[54] N. H. Alombah, H. Fotsin, and K. Romanic, "Coexistence of multiple attractors, metastable chaos and bursting oscillations in a multiscroll memristive chaotic circuit," International Journal of Bifurcation and Chaos, vol. 27, no. 5, Article ID 1750067, 2017.

[55] B. Bo-Cheng, L. Zhong, and X. Jian-Ping, "Transient chaos in smooth memristor oscillator," Chinese Physics B, vol. 19, no. 3, Article ID 030510, 2010.

[56] S. Sabarathinam, C. K. Volos, and K. Thamilmaran, "Implementation and study of the nonlinear dynamics of a memristor-based Duffing oscillator," Nonlinear Dynamics, vol. 87, no. 1, pp. 37-49, 2017.

[57] C. Grebogi, E. Ott, and J. A. Yorke, "Chaotic attractors in crisis," Physical Review Letters, vol. 48, no. 22, pp. 1507-1510, 1982.

[58] K. Otsuka, "Chaotic itinerancy in a coupled-element multistable optical chain," Physical Review A, vol. 43, no. 1, pp. 618-621, 1991.

[59] A. Chantre and L. C. Kimerling, "Configurationally multistable defect in silicon," Applied Physics Letters, vol. 48, no. 15, pp. 1000-1002, 1986.

[60] N. Wang, G. Zhang, N. V. Kuznetsov, and H. Bao, "Hidden attractors and multistability in a modified Chua's circuit," Communications in Nonlinear Science and Numerical Simulation, vol. 92, Article ID 105494, 2021.

[61] Y.-Z. Cheng, F.-H. Min, Z. Rui, and L. Zhang, "A heterogeneous dual memristive circuit: multistability, symmetry and FPGA implementation," Chinese Physics B, 2021.

[62] M. A. Al-hayali and F. S. Al-Azzawi, "A 4D hyperchaotic Sprott S system with multistability and hidden attractors," Journal of Physics: Conference Series, vol. 1879, no. 3, Article ID 032031, 2021.

[63] Q. Xu, S. Cheng, Z. Ju, M. Chen, and H. Wu, “Asymmetric coexisting bifurcations and multi-stability in an asymmetric memristive diode-bridge-based Jerk circuit," Chinese Journal of Physics, vol. 70, pp. 69-81, 2021.

[64] F. Yu, L. Liu, H. Shen et al., "Multistability analysis, coexisting multiple attractors, and FPGA implementation of Yu-Wang Four-Wing chaotic system," Mathematical Problems in Engineering, vol. 2020, Article ID 7530976, 16 pages, 2020.

[65] K. Altun, "FPAA implementations of fractional-order chaotic systems," Journal of Circuits, Systems, and Computers, Article ID 2150271, 2021.

[66] T. Liu, H. Yan, S. Banerjee, and J. Mou, "A fractional-order chaotic system with hidden attractor and self-excited attractor and its DSP implementation," Chaos, Solitons \& Fractals, vol. 145, Article ID 110791, 2021.

[67] Q. Lai, Z. Wan, P. D. Kamdem Kuate, and H. Fotsin, "Coexisting attractors, circuit implementation and synchronization control of a new chaotic system evolved from the 
simplest memristor chaotic circuit," Communications in Nonlinear Science and Numerical Simulation, vol. 89, Article ID 105341, 2020.

[68] Y. Peng, S. He, and K. Sun, "A higher dimensional chaotic map with discrete memristor," AEU-International Journal of Electronics and Communications, vol. 129, Article ID 153539, 2021.

[69] S. He, K. Sun, Y. Peng, and L. Wang, "Modeling of discrete fracmemristor and its application," AIP Advances, vol. 10, no. 1 , Article ID 015332, 2020. 